\title{
Development, Structure, and Mechanism of Synthetic Antibodies that Target Claudin and Clostridium perfringens Enterotoxin Complexes
}

\author{
Benjamin J. Orlando ${ }^{1}$, Pawel K. Dominik ${ }^{2, a}$, Sourav Roy ${ }^{3}$, Chinemerem Ogbu ${ }^{3}$, Satchal K. Erramilli ${ }^{2}$, \\ Anthony A. Kossiakoff ${ }^{2}$, and Alex J. Vecchio ${ }^{\#, 3, *}$ \\ ${ }^{1}$ Department of Biochemistry and Molecular Biology, Michigan State University, East Lansing, MI, USA \\ ${ }^{2}$ Department of Biochemistry and Molecular Biology, University of Chicago, Chicago, IL, USA \\ ${ }^{3}$ Department of Biochemistry, University of Nebraska-Lincoln, Lincoln, NE, USA \\ ${ }^{a}$ Present address: Pfizer, San Diego, CA, USA \\ ${ }^{\#}$ Lead/First author \\ ${ }^{*}$ Corresponding author: email avecchio@unl.edu
}

A.J.V. ORCID 0000-0002-4222-7874

\begin{abstract}
Strains of the Gram-positive bacterium Clostridium perfringens produce a two-domain enterotoxin $(\mathrm{CpE})$ that afflict millions of humans and domesticated animals annually by causing prevalent gastrointestinal illnesses. CpE's C-terminal domain (cCpE) binds cell surface receptors then its N-terminal domain restructures to form a membrane-penetrating $\beta$-barrel pore, which is toxic to epithelial cells of the gut. The claudin family of membrane proteins are the receptors for $\mathrm{CpE}$, and also control the architecture and function of cell/cell contacts called tight junctions that create barriers to intercellular transport of solutes. $\mathrm{CpE}$ binding disables claudin and tight junction assembly and induces cytotoxicity via $\beta$-pore formation, disrupting gut homeostasis. Here, we aimed to develop probes of claudin/CpE assembly using a phage display library encoding synthetic antigen-binding fragments (sFabs) and discovered two that bound complexes between human claudin -4 and cCpE. We established each sFab's unique modes of molecular recognition, their binding affinities and kinetics, and determined structures for each sFab bound to $\sim 35 \mathrm{kDa}$ claudin-4/cCpE in three-protein comprised complexes using cryogenic electron microscopy (cryoEM). The structures reveal a recognition epitope common to both sFabs but also that each sFab distinctly conforms to bind their antigen, which explain their unique binding equilibria. Mutagenesis of antigen/sFab interfaces observed therein result in further binding changes. Together, these findings validate the structures and uncover the mechanism of targeting claudin- $4 / \mathrm{cCpE}$ complexes by these sFabs. Based on these structural insights we generate a model for CpE's cytotoxic claudin-bound $\beta$-pore that predicted that these two sFabs would not prevent $\mathrm{CpE}$ cytotoxicity, which we verify in vivo with a cell-based assay. This work demonstrates the development and targeting mechanisms of sFabs against claudin/cCpE that enable rapid structural elucidation of these small membrane protein complexes using a cryoEM workflow. It further provides a structure-based framework and therapeutic strategies for utilizing these sFabs as molecular templates to target claudin/ $\mathrm{CpE}$ assemblies, obstruct $\mathrm{CpE}$ cytotoxicity, and treat $\mathrm{CpE}$-linked gastrointestinal diseases that cause substantial economic and quality of life losses throughout the world.
\end{abstract}

\section{INTRODUCTION}

Tight junctions (TJs) are molecular gatekeepers that regulate transport of small molecules through the paracellular spaces between adjoining cells in endothelia and epithelia. To accomplish this function, TJs possess integral membrane proteins that self-assemble to simultaneously span both intracellular and paracellular spaces $(1, \underline{2})$. Of the numerous membrane proteins at TJs, the 27 -member family of claudins comprise the major structural and function backbone of TJs, making them attractive targets to modulate TJ barriers therapeutically ()ㅡ. Evolution has successfully accomplished this feat. Type F strains of the pathogenic Gram-positive bacterium Clostridium perfringens produce an enterotoxin $(\mathrm{CpE})$ that binds claudins to dissociate TJs during cytotoxicity in the gut (4-6). In domesticated animals, CpE causes necrotic enteritis, colitis, and diarrhea $(\underline{7}, \underline{8})$. In humans $\mathrm{CpE}$ causes enterotoxemia; is the $3^{\text {rd }}$ most prevalent 
foodborne illness in the United States causing an estimated $\sim \$ 400$ million annual economic burden; and is the source of a further 4+ million food poisoning cases worldwide-some resulting in death ( 7, 9-12). Unlike other human diseases caused by Clostridium perfringens toxins, $\mathrm{CpE}$-associated ailments are not directly preventable nor treatable, and no vaccine exists against this food poisoning type (13). Because $\mathrm{CpE}$ is heat-resistant and produced by spore-formed Clostridium perfringens, cooking does little to reduce its pathogenicity (14). Therefore, sub-molecular details into CpEs mechanisms of claudin binding and dissociating $\mathrm{TJ}$ barriers are essential to elucidate $\mathrm{CpE}$ cytotoxicity and to develop therapeutic strategies for CpE-based diseases.

The C-terminal domain of $\mathrm{CpE}(\mathrm{cCpE}$ ) selectively targets claudins in the gut by recognizing a motif unique to these receptors, then binds them with low nanomolar affinities $(\underline{15}, \underline{16})$. This claudin-bound $\mathrm{CpE}$, i.e. "small complex", then oligomerizes and its N-terminal domain structurally rearranges to form a membranepenetrating and cytotoxic $\beta$-barrel pore $(\underline{17}, \underline{18})$. The process of $\beta$-pore formation disables claudin/claudin interactions vital to TJ assembly and ultimately dissociates TJs causing paracellular leakage prior to CpEinduced cell death. Crystal structures of $\mathrm{cCpE}$ bound to receptor claudins have shed light on their interprotein interactions and have helped to inform structure-guided design of modified cCpEs used to detect or destroy cancer cells or to modulate the blood-brain barrier for drug delivery (19-27). Yet, a complete structural and mechanistic understanding of $\mathrm{CpE}$ dissociation of TJs and the process of cytotoxic $\beta$-pore formation remains elusive.

We intended to elucidate how $\mathrm{CpE}$ binds claudins and dissociates TJs by determining X-ray crystal structures of enterotoxins $\mathrm{CpE}$ or $\mathrm{cCpE}$ in complex with claudins but found crystallization to be a bottleneck. For most claudins, crystals did not form at all, while for those that formed crystals it required screening and optimizing hundreds over $\sim$ one year to determine structures resolved to 3-4 $\AA(22, \underline{23})$. Using a phage display library encoding synthetic antigen-binding fragments (sFabs) we sought to discover molecules that target and bind complexes between enterotoxins and human claudin-4 (claudin-4). Our goal was to use sFabs to chaperone crystallization, improve initial diffraction, and increase structural throughput of this and other claudin/enterotoxin complexes $(\underline{28}, \underline{29})$. Through this approach, we surmised, additional sFabs could be discovered that obstruct complex formation altogether and be useful in therapeutic development. During this process three sFabs were discovered, which we termed $\underline{\mathrm{C}} \mathrm{pE}$ Obstructing Proteins (COPs). Preliminary characterization of COPs revealed that COP-2 and COP-3 had properties amenable for structure determination of the claudin $-4 / \mathrm{cCpE}$ complex. Ultimately, however, we determined a structure of this complex using a traditional crystallography workflow (23). But because sFabs have recently been shown effective for determining structures of small membrane proteins and complexes by cryogenic electron microscopy (cryoEM), we used COP-2, COP-3, and cryoEM to progress a novel workflow for higher throughput elucidation of claudin/enterotoxin structures (30-32).

Here, we qualitatively and quantitatively characterize COP-2 and COP-3 binding to claudin-4, cCpE, and $\mathrm{CpE}$ individually, and to claudin-4/enterotoxin complexes using biochemical and biophysical techniques. We also use cryoEM at $200 \mathrm{keV}$ to determine 4-7 $\AA$ structures of each $50 \mathrm{kDa}$ COP bound to $\sim 35 \mathrm{kDa}$ claudin-4/cCpE complexes in one month; and employ these structures to create models of the cytotoxic $\mathrm{CpE} \beta$-pore. Our findings reveal the structural basis and COP-specific mechanisms of COP targeting of claudin-4/cCpE complexes. This research independently validates claudin-4/cCpE structures determined by X-ray crystallography and provides a structural framework for preventing CpE-mediated cytotoxicity by obstructing toxin/receptor binding. Moreover, it advances development of technologies and establishes a general approach for determining structures of other claudin/enterotoxin complexes at moderate resolutions rapidly using practical cryoEM instrumentation that can readily be expanded to higher resolutions with $300 \mathrm{keV}$ microscopes. Further, it demonstrates the antigenicity of $\mathrm{CpE}$ and $\mathrm{cCpE}$ enterotoxins and their claudin-bound complexes, which through intensified sFab development could generate novel sFabs useful for modulating TJ barriers or as therapeutics for preventing or treating $\mathrm{CpE}$ based illnesses in humans and domesticated animals. 


\begin{abstract}
RESULTS
Development of COPs. claudin-4 solubilized in n-dodecyl- $\beta$-D-maltopyranoside (DDM) and bound to $\mathrm{cCpE}$ was used as input for phage display selection using a large and diverse library of sFabs based on a humanized Fab scaffold (see Methods). The sFab library has varied sequences that are biased for serine and tyrosine in the complimentarity-determining regions (CDRs) of their light (L) and heavy (H) chains within variable domains $(\underline{33}, \underline{34})$. After several rounds of selection to increase stringency, two sFabs, termed COP-2 and COP-3, were further developed and validated by ELISA to bind to claudin-4/cCpE. Both COPs were sequenced, recombinantly expressed in E. coli, and purified using affinity chromatography in order to isolate these sFabs and characterize their binding further. Sequence alignments of COP-2 and COP-3 reveal their unique primary sequences (SI Appendix, Fig. S1). The alignments show that COP-2 and COP-3 share 98.2 and $93.3 \%$ sequence identity in their L and $\mathrm{H}$ chains, respectively. The COP sequences diverge the greatest in CDR-L3, CDR-H1, and CDR-H3. These residue divergences may direct COP-specific recognition of claudin-4/cCpE complexes.
\end{abstract}

Biochemical Characterization of COPs. To obtain more detailed insights into COP recognition we determined which molecule COPs bind and if they use unique or common epitopes. After expressing and purifying claudin- $4, \mathrm{c} \mathrm{CE}, \mathrm{CpE}$, and $\mathrm{COPs}$, increases in molecular masses as assessed by decreases in peak retention times with size-exclusion chromatography (SEC) was used to qualitatively characterize COP-2 and COP-3 binding modes (see Methods). We observed, after incubating COP-2 and COP-3 with claudin4 and $\mathrm{cCpE}$ alone, that COPs did not bind claudin-4 but did form larger complexes with cCpE (SI Appendix, Fig. S2A and S2B). Incubating claudin-4, cCpE, and COPs together showed that $\mathrm{cCpE}$ binds claudin-4 and that both COPs bound claudin-4/cCpE complexes (SI Appendix, Fig. S2C). No mass increases were observed for COPs incubated with CpE (SI Appendix, Fig. S2D). Lastly, incubating claudin-4, CpE, and COPs together showed that while CpE binds claudin-4 and forms a "small complex", the COPs do not bind "small complexes" (SI Appendix, Fig. S2E). To verify complex formation, peaks from SEC were pooled and subjected to SDS-PAGE, which showed the presence of individual proteins from associated complexes (SI Appendix, Fig. S2F). To determine if COP-2 and COP-3 share a binding epitope, we incubated both together with $\mathrm{cCpE}$. If COPs bound distinct epitopes a molecular mass shift greater than the individual $\mathrm{cCpE} / \mathrm{COP}$ complex would result. SEC revealed no additive mass shift with both COPs present (SI Appendix, Fig. S2B). These biochemical results suggest the specific molecular recognition of COPs.

Biophysical Characterization of COPs. After qualitatively establishing COP binding we quantitated the affinities and kinetics of COP interactions with claudin-4 and enterotoxins. We determined the secondorder association rate constant $\left(\mathrm{k}_{\mathrm{on}}\right)$, first-order dissociation rate constant $\left(\mathrm{k}_{\mathrm{off}}\right)$, and equilibrium dissociation constant $\left(\mathrm{K}_{\mathrm{D}}\right)$ of these interactions using bio-layer interferometry (BLI) (Table 1). BLI measurements were made using pre-formed claudin-4/enterotoxin complexes or $\mathrm{cCpE}$ alone in DDM, replicating the conditions of the phage display selections. For COP-2 and COP-3 binding to claudin- $4 / \mathrm{cCpE}$ complexes we measured $\mathrm{K}_{\mathrm{Ds}}$ of 52.3 and $98.4 \mathrm{nM}$, respectively. Comparing the binding rates revealed that COP-3 had 1.9- and 3.5fold faster $\mathrm{k}_{\mathrm{on}}$ and $\mathrm{k}_{\text {off }}$ rates compared to COP-2. The $\mathrm{K}_{\mathrm{Ds}}$ of COP-2 and COP-3 to cCpE were 67.6 and $137.6 \mathrm{nM}$, respectively. Like COP-3 binding to claudin-4/cCpE complexes, the $\mathrm{k}_{\text {on }}$ and $\mathrm{k}_{\text {off }}$ rates were 1.4and 2.1-fold faster when compared to COP-2. Finally, we measured $\mathrm{K}_{\mathrm{Ds}}$ of 7.8 and $9.1 \mu \mathrm{M}$ for COP-2 and COP-3 binding to claudin-4/CpE "small complexes", respectively. The $\mathrm{K}_{\mathrm{D}}$ values represent 149.1- and 92.0fold decreases in COP-2 and COP-3 affinity for CpE compared to cCpE. Kinetic differences in $\mathrm{k}_{\text {on }}$ and $\mathrm{k}_{\text {off }}$ of COP binding are visible in the BLI sensorgrams (Fig. 1B, 2B, and SI Appendix, Fig. S3). These results agree with biochemical assessment and reveal biophysical parameters unique to each COP that may influence recognition and binding to claudin-4/cCpE complexes.

CryoEM Structure of claudin-4/cCpE/COP-2 Complex. Having confirmed that COP-2 binds claudin4/cCpE complexes using SEC (Fig. 1A) and BLI (Fig. 1B), we next delineated the structural basis and molecular mechanism of COP-2 targeting by determining its structure by cryoEM. CryoEM screening of 
claudin-4/cCpE/COP-2 complexes that were SEC purified, pooled, and concentrated to $8.0 \mathrm{mg} / \mathrm{mL}$ in various detergents showed that those solubilized in 2,2-didecylpropane-1,3-bis- $\beta$-D-maltopyranoside (LMNG) were amenable to structure determination. This was confirmed by subsequent $2 \mathrm{D}$ classifications and $a b$ initio 3D reconstructions, which revealed a stacked and linear arrangement of the three proteins, a canonical two-lobed sFab, and flexibility in COP-2's constant domain (SI Appendix, Fig. S4). Due to this flexibility, we used a data processing strategy that masked claudin-4, cCpE, and the variable domains of COP-2 to resolve and focus understanding on the interactions directing COP-2 recognition. Using this strategy, we generated a final processed cryoEM map that was resolved to $6.9 \AA$ (SI Appendix, Table S1). The map resolution was sufficient to reveal the claudin-4/cCpE/COP-2 complex, secondary structural elements including claudin-4's four transmembrane helices (TM), and some bulky side chains (SI Appendix, Fig. S5A). This cryoEM map was used to build, refine, and determine a structure for the claudin4/cCpE/COP-2 complex. SI Appendix, Table S1 shows data processing, refinement, and model-to-map fit statistics, with further details given in Methods.

The cryoEM map resolution was insufficient to place side chains confidently, so we cannot verify interactions between claudin- 4 and cCpE that define the "cCpE-binding motif" (23). Overall, however, the claudin-4/cCpE portion of this complex from cryoEM superimposes well onto the crystal structure (SI Appendix, Fig. S6A). We measured root mean square deviations (RMSDs) in C $\alpha$ positions of 2.0 and 1.4 $\AA$ between the cores of claudin- 4 and cCpE, and $1.7 \AA$ in overall secondary structures between the cryoEM and crystal structures of the claudin- $4 / \mathrm{cCpE}$ complex, indicating no major conformational changes occur upon COP-2 binding. Generally, the claudin-4 extracellular segments (ECS) are in similar conformations and thus may interact with cCpE similarly in the cryoEM and crystal structures (SI Appendix, Fig. S6A). Density corresponding to a loop within ECS2, which contains the NPLVA ${ }^{153}$ motif shows that the motif accesses a groove on the surface of $\mathrm{cCpE}$. This interaction is known to impart high-affinity cCpE binding to claudins and COP-2 appears to not significantly alter its structure (23).

The cryoEM structure of the claudin- $4 / \mathrm{cCpE} / \mathrm{COP}-2$ complex reveals the basis of COP-2 binding. The canonical binding of $\mathrm{cCpE}$ to claudin-4 exposes cCpE's top half to COP-2 binding by providing an antigenic surface (Fig. 1C). COP-2 binding to $\mathrm{cCpE}$ alters the conformations of $\mathrm{cCpE}$ 's N-terminus, $\beta$ strands $\beta 5$ and $\beta 6$, and the loop connecting them, when compared to the crystal structure (SI Appendix, Fig. S6A). COP-2's L chain sits atop a depression on the exterior of cCpE formed between the N-terminus and strands $\beta 5$ and $\beta 6$ (Fig. 1C). Chain L's CDR-L1, -L2, and -L3 conform to the surface of cCpE and potential side chains involved in these interactions can be visualized although not placed confidently (Fig. 1D and 1E). COP-2's chain $H$ access the same surface depression as chain $L$ but also flanks the opposite side of strands $\beta 5$ and $\beta 6-$ CDR-H3 shares an epitope with chain L while CDR-H1 and -H2 reside on the other (Fig. 1C and 1F). CDR-H3 splays outward to deeply penetrate its surface groove, conforming to the cCpE surface (Fig. 1G). Based on the interactions projected by the structure, we hypothesized that residues comprising Lys 197 to Leu202 and Asn267 to Gln276 in cCpE could influence COP-2 binding.

CryoEM Structure of claudin-4/cCpE/COP-3 Complex. After verifying that COP-3 binds claudin4/cCpE complexes using SEC (Fig. 2A) and BLI (Fig. 2B), we next determined a structure for COP-3 in complex with claudin-4/cCpE by cryoEM to contrast its molecular mechanism of targeting with COP-2. claudin-4/cCpE/COP-3 complexes in various membrane mimetics were SEC purified, pooled, and concentrated to $6.0 \mathrm{mg} / \mathrm{mL}$ for cryoEM (see Methods). Those complexes solubilized in amphipol were superior to LMNG and DDM for cryoEM based on 2D class averages. The 2D and 3D classifications of the COP-3 complex showed a single complex with each protein stacked in a linear arrangement and had features corresponding to secondary structural elements and both lobes of COP-3, indicating that maps of adequate resolution were obtained (SI Appendix, Fig. S7). The 3D reconstructions showed that the constant domains of COP-3 are less dynamic than those of COP-2, but also that claudin-4's TM region was less well resolved in amphipol. We used a data processing strategy that masked COP-3 to best resolve the interactions directing its recognition of claudin- $4 / \mathrm{cCpE}$, which generated a final processed map (focused) that was 
resolved to $3.8 \AA$ and showed structural features including density for bulky side chains (SI Appendix, Table S1). However, this map lacked definition in the $\mathrm{cCpE}$ and claudin-4 regions, and optimization of the model-to-map fit proved difficult. Therefore, data was processed with no mask, which produced a final map (whole) that was resolved to $5.0 \AA$. The whole map resolved the claudin- $4 / \mathrm{cCpE} / \mathrm{COP}-3$ complex, including secondary structural elements like claudin-4's TMs and $\alpha$-helices and $\beta$-strands in cCpE, and the conformations of COP-3 CDRs (SI Appendix, Fig. S5B). Initial model building and refinement employed both maps, but the final model was refined against the whole map, resulting in the cryoEM structure for the claudin-4/cCpE/COP-3 complex. SI Appendix, Table S1 shows data processing, refinement, and modelto-map fit statistics, with further details given in Methods.

The cryoEM structure of the claudin-4/cCpE/COP-3 complex reveals COP-3's mode of binding. Compared to the claudin $-4 / \mathrm{cCpE}$ crystal structure, the equivalent portion from the COP-3 cryoEM structure superimposes well and indicates that COP-3 does not induce large conformational changes or affect normal claudin-4/cCpE interactions (SI Appendix, Fig. S6B). We measured RMSDs in C $\alpha$ positions of 2.2 and 1.2 $\AA$ between the cores of claudin- 4 and cCpE, and $2.1 \AA$ between overall secondary structures in the claudin$4 / \mathrm{cCpE}$ complex when comparing cryoEM and X-ray structures. Like COP-2, COP-3 accesses cCpE's surface opposite to where claudin-4 binds due their canonical interactions (Fig. 2C). COP-3 binding to $\mathrm{cCpE}$ alters cCpE's N-terminus, $\beta 5$ and $\beta 6$, and the loop connecting them, when compared to the crystal structure (SI Appendix, Fig. S6B). The L chain of COP-3 binds between cCpE's N-terminus and $\beta 5$ and $\beta 6$ using CDR-L1 and CDR-L3 (Fig. 2D). The CDR-L3 loop conforms to the surface of this region, potentially using aromatic side chains to drive shape complementarity (Fig. 2E). COP-3's H chain flanks both sides of $\beta 5$ and $\beta 6$ of $\mathrm{cCpE}$ with CDR-H3 sharing an epitope with chain L while CDR-H1 and CDRH2 occupy the other side (Fig. 2C and 2F). COP-3's CDR-H3 conforms to and deeply accesses a surface groove between this region and the N-terminus (Fig. 2G). Based on the interactions approximated by the structure, we hypothesized that residues comprising Glu198 to Leu202 and Asn269 to Gln276 in cCpE may guide COP-3 binding.

Comparison of COP Structures. Because structure resolution was limiting we used computation-based structure and sequence analyses to estimate the $\mathrm{COP}$-specific residues used for $\mathrm{cCpE}$ recognition. For this, we input our structures into PDBePISA, an online tool for determining the structural and chemical properties of macromolecular interfaces, and then compared areas determined by PDBePISA to preside over cCpE/COP binding with COP primary sequences (ㅎ5) (SI Appendix, Fig. S1). For COP-2 we found that CDR-L3 residues Tyr117 to Ala120, CDR-H1 residue Ser56, and CDR-H3 residues Tyr125 to Ser137 were unique to COP-2. For COP-3 we found that CDR-L3 residues His117 to Tyr121, CDR-H1 residue Tyr56, and CDR-H3 residues Gly125 to Tyr137 were unique to COP-3. When compared to a generic sFab, COPs contain many aromatic side chains and are enriched in serine and tyrosine residues in their CDRs (SI Appendix, Fig. S1). This analysis exposes potential COP amino acid determinants for recognition and binding of claudin-4/cCpE complexes.

To contrast COP binding modes and to explain their varied biophysical binding equilibria, we overlaid our two cryoEM structures (SI Appendix, Fig. S8). The overlays revealed that: 1) the cCpE poses when bound to claudin-4 are similar but structural perturbations exist in $\mathrm{cCpE}$ due to $\mathrm{COP}$-induced changes; 2) the claudin-4/cCpE portions exhibit minor structural differences between complexes but the claudin-4 TMs are oriented differently in membrane mimetics; and 3) the COPs have similar secondary structural elements, but their tertiary structures and CDRs vary in conformations (SI Appendix, Fig. S8A). Focusing on regions with the largest observable differences, we found that the $\mathrm{L}$ and $\mathrm{H}$ chains of each $\mathrm{COP}$ conform to $\mathrm{cCpE}$ uniquely. For COP-2 chain L, CDR-L1 and CDR-L3 reside within to the surface groove formed between the N-terminus and $\beta 5-\beta 6$ of cCpE while CDR-L2 lies external (SI Appendix, Fig. S8B). For COP-3 chain $\mathrm{L}$, the surface groove is depressed due to less N-terminal length, so while CDR-L1 and CDR-L3 reside in the same groove, CDR-L1 appears to interact less with cCpE. The conformation of CDR-H3 may force this CDR-L1 change in COP-3. Using PDBePISA we calculated interface surface areas between the L chain of 
COPs and cCpE and found that COP-2's area was 36.4\% larger. In both COP's chain H, the CDR-H1 and CDR-H2 bind on one side of cCpE's $\beta 5-\beta 6$ element while CDR-H3 flanks the other (SI Appendix, Fig. S8C). CDR-H1 and CDR-H2 have similar conformations and sequence alignments show that residue conservation is high here (SI Appendix, Fig. S1). But CDR-H3 conformations appear to vary and sequences also show the greatest diversity exists here. Generally, however, the conformations of all three CDRs overlay well between COPs, which is reflected by $\mathrm{cCpE} / \mathrm{H}$ chain interface surface area differences of only $4.5 \%$. For both COPs, $\sim 80 \%$ of the total $\mathrm{cCpE} / \mathrm{COP}$ interface area resides on chain H. Overall, COP-2 appears to employ chain L to a higher degree than COP-3, while both COPs use chain H similarly and more dominantly to chain $\mathrm{L}$ to bind $\mathrm{cCpE}$. Moreover, the surface structure of $\mathrm{cCpE}$ appears to be changed more by COP-2 than COP-3 as a result of binding, indicating that COP-specific interactions, driven by sequence diversity, may uniquely mold cCpE's surface (SI Appendix, Fig. S8B and S8C). We next determined whether the structural observations detailed here were true in vitro and if they could explain the measured differences in binding equilibria between COP-2 and COP-3.

Quantification of COP Binding to Mutant cCpEs. To test our structures and pinpoint the amino acid determinants of COP binding we mutated regions of $\mathrm{cCpE}$ where we observed potential interactions with COPs and quantified binding. Table 2 shows sequences for $\mathrm{cCpE}^{\text {mutants }}$ that were generated. Pre-formed claudin-4/cCpE $\mathrm{Cp}^{\text {mutant }}$ complexes were used to mimic the $\mathrm{sFab}$ selection experiment. First, we found that no $\mathrm{cCpE}^{\text {mutant }}$ affected binding to claudin-4, which binds with a $\mathrm{K}_{\mathrm{D}}$ of $2.5 \mathrm{nM}$ (SI Appendix, Fig. S3E) (23, 36). Next, we tested COP binding to claudin-4/cCpE ${ }^{\text {mutant }}$ complexes using BLI (Table 3). Binding measurements showed that mutant $\mathrm{cCpE}^{1}$, which has a shortened N-terminus, decreased affinity for COP2 and COP-3 primarily by increasing $\mathrm{k}_{\text {off }}$ rates. Mutant $\mathrm{cCpE}^{2}$ increased COP-2 and COP-3 affinities through increased $\mathrm{k}_{\text {on }}$ rates - this mutation lies at the end of the N-terminus at the start of globular cCpE. Mutant $\mathrm{cCpE}^{2}$ with an added Leu202Ala mutation, $\mathrm{cCpE}^{2 \mathrm{~L}}$, showed binding to both COP-2 and COP-3 at near $\mathrm{cCpE}^{\text {wild type }}$ affinities, indicating a loss of affinity compared to mutant $\mathrm{cCpE}^{2}$ due to the shorter alanine side chain. Kinetics reveal that COPs bind claudin- $4 / \mathrm{cCpE}^{2 \mathrm{~L}}$ with slower $\mathrm{k}_{\text {on }}$ and faster $\mathrm{k}_{\text {off }}$ rates compared to claudin-4/cCpE ${ }^{2}$. The mutant $\mathrm{cCpE}^{3}$ in the loop connecting $\beta 5$ to $\beta 6$ deceased affinity for COP-2 by 1.2 fold - but for COP-3 it deceased affinity 12.4-fold - this greater affinity loss for COP-3 is driven by a 14.4fold faster $\mathrm{k}_{\text {off }}$ rate. Both COPs exhibited substantial losses in affinity to mutant $\mathrm{cCpE}^{4}$, which alters residues in the $\beta 5$ to $\beta 6$ connecting loop and $\beta 6$ to alanine. COP-2 affinity decreased 4,835.6-fold while COP-3 decreased 135.2-fold. Unlike other $\mathrm{cCpE}$ mutants, $\mathrm{cCpE}^{4}$ affected both $\mathrm{k}_{\text {on }}$ and $\mathrm{k}_{\text {off }}$ rates, indicating that this mutant radically perturbed normal COP binding. To put these results in a structural context, we made models of mutant cCpEs based on our cryoEM structures in order to compare them to cCpE ${ }^{\text {wildtype }}$ (SI Appendix, Fig. S9). These models provide structural bases for COP binding to mutant cCpEs that explain our biophysical measurements. Overall, each set of cCpE mutants, chosen based on their potential for side chain interactions with COPs, altered COP binding in different ways, providing in vitro validation of our structures.

Model of Cytotoxic Claudin-bound CpE Pore. To predict COP function in the context of CpE-induced cytotoxicity, we created models for the process of COP binding to the claudin- $4 / \mathrm{CpE}$ "small complex" and for a claudin-4-bound $\mathrm{CpE} \beta$-barrel pore complex, using our cryoEM structures as guides (see Methods). We first modeled $\mathrm{CpE}$ binding to claudin-4 then COP recognition of the "small complex" (Fig. 3A). The model shows that if $\mathrm{CpE}$ is bound to claudin-4 in a "small complex" then its N-terminal domain would sterically shield COPs from access to their binding epitopes on $\mathrm{cCpE}$. This finding explains results we obtained from biophysical measurements (Table 1). We next modelled the claudin-4-bound CpE $\beta$-pore complex (Fig. 3B). As there is no structure for the $\mathrm{CpE} \beta$-pore, we used the cryoEM structure of lysenin, a $\beta$-pore toxin with homology to $\mathrm{CpE}$ to model it (37). Lysenin was chosen because attempts to model the complex using the aerolysin $\beta$-pore, another homologous protein, did not place claudin perpendicular to the membrane plane. We thus hypothesize that the $\mathrm{CpE} \beta$-pore resembles lysenin more than aerolysin. This model reveals that when oligomeric $\mathrm{CpE}$ assembles into a $\beta$-pore complex that $\mathrm{COP}$ access to $\mathrm{c} \mathrm{CpE}$ binding 
epitopes would be sterically obstructed even if CpE N-terminal domain rearrangements occur (Fig. 3B and 3C). These structural models explain our in vitro binding data and provide a prediction for the effect of COPs on CpE-induced cytotoxicity in vivo.

Effect of COPs on CpE Cytotoxicity. Finally, we used a cell-based assay to validate our model of the claudin-4-bound $\mathrm{CpE} \beta$-pore and to test whether COPs affect CpE-induced cytotoxicity (see Methods). Using Spodoptera frugiperda cells that lack endogenous claudins but form tight junction-like strands when expressing claudin- 4 on their cell surfaces, we added COP-2 or COP-3, followed immediately by cCpE or $\mathrm{CpE}$, using previously described methods $(22, \underline{23}, \underline{38}, \underline{39})$. Control wells consisted of no addition of COPs or enterotoxins. We then measured cell viability by quantifying the amount of cell death instigated by enterotoxins to determine whether COPs altered cytotoxicity (Fig. 3C). For cells expressing claudin-4 alone not treated with COPs we found that cell viability averaged $73.5 \%$. For these cells, COP addition decreased average viability by $0.7 \%$, indicating no COP-induced cytotoxicty. For cells expressing claudin- 4 and treated with $\mathrm{cCpE}$, which lacks the cytotoxic N-terminus, we found that cell viability averaged $69.7 \%$. Again, for cCpE-treated cells COP addition decreased average viability only $0.8 \%$. Finally, for cells expressing claudin-4 treated with $\mathrm{CpE}$ we found that cell viability averaged $31.3 \%$, a decrease of $40.3 \%$ compared to untreated and $\mathrm{cCpE}$-treated cells, indicating CpE-induced cytotoxicity. Addition of COPs to $\mathrm{CpE}$-treated cells did not significantly change $\mathrm{CpE}$-induced cell death, decreasing average cell viability by $4.3 \%$. These results validate our in silico model of the claudin-4-bound $\mathrm{CpE} \beta$-pore and establish the effect of COPs to CpE-induced cytotoxicity.

\section{DISCUSSION}

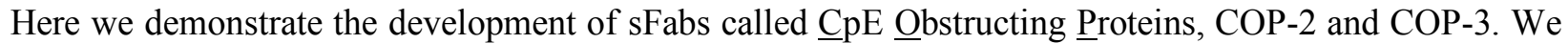
show that COPs bind well to cCpE but not to claudin- 4 or $\mathrm{CpE}$; that COPs bind $\mathrm{cCpE}$ better when bound to claudin-4 than when alone in solution; and that both COPs bind the same surface epitope on $\mathrm{cCpE}$ opposite to where claudin-4 binds (SI Appendix, Fig. S2). We also show that COP binding to claudin$4 / \mathrm{cCpE}$ complexes yield similar affinities but different kinetics, with COP-2 associating and dissociating more slowly than COP-3 (Table 1). As COPs target and bind $\mathrm{cCpE}$ on the opposing surface to its claudin binding motif they are therefore capable of binding to any claudin/cCpE complex, making them a general yet strategic tool for enabling structures of claudin/cCpE complexes.

As proof of this concept, we determine cryoEM structures for COP-2 and COP-3 bound to claudin-4/cCpE complexes, which reveal the $\mathrm{c} C \mathrm{pE}$ binding epitope and the potential interaction interfaces for the $\mathrm{L}$ and $\mathrm{H}$ chains of COPs (Figs. 1 and 2). Our structures and computational analyses show that COP-2 has an 11\% larger cCpE/COP interface area, that COP-2 uses chain L CDR-L1 uniquely, and that its binding conforms to or molds cCpEs surface to a greater extent than COP-3, which explains its higher affinity and slower association and dissociation rates compared to COP-3. These analyses also reveal a common binding property to COPs where chain $\mathrm{H}$ interacts with both sides of a $\beta 5-\beta 6$ epitope in a mechanism similar to a caliper brake on a bicycle (Figs. 1G and 2G). Holistically, the cryoEM structures resemble and thus provide independent validation of the claudin-4/cCpE complex crystal structure (SI Appendix, Fig. S6).

To validate these moderate resolution cryoEM structures we mutate the observed COP binding epitopes on $\mathrm{cCpE}$ and quantify mutant effects to COP binding. For this we mutated side chains in sequential three to five residue zones, rather than making single point mutations (Table 2). We show that all mutations made and tested affect $\mathrm{cCpE} / \mathrm{COP}$ binding affinity, kinetics, or both (Table 3). Some, like mutants $\mathrm{cCpE}^{3}$ and $\mathrm{cCpE}^{4}$, display COP-specific differences in their binding and thus pinpoint residues of $\mathrm{cCpE}$ that are uniquely recognized by either COP-2 or COP-3. The changes to COP binding by cCpE mutants in vitro validates our cryoEM structures by attesting to the accuracy of their modeled $\mathrm{cCpE} / \mathrm{COP}$ interfaces. We further show that models of mutant $\mathrm{cCpE} / \mathrm{COP}$ complexes guided by our structures elucidate the structural bases of mutant effects to COP binding (SI Appendix, Fig. S9). In sum, these results provide the likely 
amino acid determinants and biophysical interaction mechanisms that direct COP-specific recognition of and binding to $\mathrm{cCpE}$.

We further present models for the claudin- $4 / \mathrm{CpE}$ "small complex" and claudin-4-bound $\mathrm{CpE} \beta$-pore complex in order to understand our structures in a functional context and to predict COP efficacy for use as $\underline{\mathrm{C}} \mathrm{pE}$ Obstructing Proteins (Fig. 3). One model shows that when $\mathrm{CpE}$ is bound to claudin-4 in a "small complex" that the N-terminus of $\mathrm{CpE}$ sterically shields COP binding epitopes and prevents binding (Fig. 3A). This model is verified by our finding that COP-2 and COP-3 bound $\mathrm{CpE}$ with $\sim 100$-fold lower affinity than cCpE (Table 1). Using the homologous $\beta$-pore-forming toxin lysenin as a benchmark, we also show how $\mathrm{CpE}$ may oligomerize to form its plasma membrane-spanning $\beta$-pore upon binding to claudin-4 (Fig. 3B). This model shows that COPs are sterically occluded from accessing their surface epitopes on cCpE due to $\mathrm{CpE}$ oligomeric assembly and $\mathrm{N}$-terminal $\beta$-pore engagement (Fig. 3C). Based on this model we hypothesize that both COP-2 and COP-3 would be ineffective at preventing CpE-induced cytotoxicity. We test this hypothesis using a cell-based cytotoxicity assay and show that cell media supplemented with COPs do not prevent nor alter cytotoxicity induced by $\mathrm{CpE}$ (Fig. 3C). This finding verifies our hypothesis and provides evidence that our model of the $\mathrm{CpE} \beta$-pore is potentially useful to predict the functional effects of structural changes to $\mathrm{CpE}$ upon binding claudin- 4 and forming a cytotoxic $\beta$-pore.

Based on these data and results we propose a framework for using COP-like molecules to target and obstruct $\mathrm{CpE}$ cytotoxicity (Fig. 4). We envision three Strategies based on the proposed sequence of events that lead to $\mathrm{CpE} \beta$-pore formation $(\underline{17}, \underline{18})$. These include developing COPs that: 1$)$ obstruct formation of claudin4/CpE "small complexes"; 2) stabilize the native fold of CpE's N-terminus to prevent its structural rearrangement that induces cytotoxicity via $\beta$-pore formation; and 3 ) obstruct $\mathrm{CpE}$ oligomerization prior to $\beta$-pore assembly. Using our models for the "small" and claudin-4-bound CpE $\beta$-pore complexes we identify five areas where targeted binding by $\mathrm{COP}$-like sFabs could obstruct $\mathrm{CpE}$ binding, conformational changes, or oligomerization, potentially preventing $\mathrm{CpE}$-based cytotoxicity. Area $\mathrm{A}$ is a surface pocket between $\beta 8$ and $\beta 9$ on the $\mathrm{cCpE}$ domain of $\mathrm{CpE}$ (Fig. 4A); and Area B comprises the two ECS of claudins and includes the NPLVA ${ }^{153}$ motif that imparts high-affinity CpE binding (Fig. 4B) (23). Development of COPs that bind Area A or B would inhibit formation of the "small complex" by obstructing areas known to facilitate claudin-4/cCpE interactions, thus preventing $\mathrm{CpE}$ pre-pore and $\beta$-pore complex formation (Strategy 1). Area $\mathrm{C}$ is a region of $\mathrm{CpE}$ 's $\mathrm{N}$-terminus that we hypothesize structurally rearranges upon $\mathrm{CpE}$ assembly, which, like lysenin, then forms a long, antiparallel $\beta$-barrel to create the membrane-penetrating $\beta$-pore (Figs. 3B and 4C). Discovery of COPs that bind Area C may stabilize CpE's N-terminus to disable its $\beta$ pore-forming conformational transition (Strategy 2) - they may also obstruct $\mathrm{CpE}$ oligomerization (Strategy 3). Finally, Area D includes strand $\beta 1$ and helix $\alpha 1$ of $\mathrm{cCpE}$; while Area E comprises two loops that connect $\beta 2$ to $\beta 3$ and $\beta 8$ to $\beta 9$ (Fig. 4C). Based on our models, Areas D and E also represent parts of $\mathrm{cCpE}$ where $\mathrm{COP}$ binding would sterically obstruct $\mathrm{CpE}$ oligomerization. In summary, our models reveal that: 1) for COP binding to Areas A or B to be effective at obstructing cytotoxicity they will need to be present before or during $\mathrm{CpE}$ production to prevent "small complex" formation; and 2) COP binding to Areas C, D, or E would not prevent "small complex" formation and thus could be effective post-production of $\mathrm{CpE}$ but would need to be present prior to $\mathrm{CpE}$ 's oligomeric assembly of its $\beta$-pore. This proposed structure-based framework can inform therapeutic strategies that utilize unyet developed COPs to target $\mathrm{CpE}$, claudin-4, or claudin- $4 / \mathrm{CpE}$ complexes. COPs with traits that enable recognition of the abovementioned Areas could obstruct $\mathrm{CpE} \beta$-pore formation and thus prevent $\mathrm{CpE}$-linked cytotoxicity. Such molecules would be potent therapeutics, capable of treating gastrointestinal illnesses linked to $\mathrm{CpE}$ that annually afflict millions of humans and domesticated animals globally, resulting in large economic burdens and losses in quality of life. 
In this study we progress a novel workflow for rapid determination of claudin/enterotoxin structures using practical $200 \mathrm{keV}$ cryoEM instrumentation that yields 4-7 $\AA$ resolutions. The approach appears amenable to other claudins in a variety of membrane mimetics, and is enabled by $50 \mathrm{kDa}$ COPs that add mass, rigidity, and act as fiducial marks for cryoEM (SI Appendix, Fig. S10). The COPs are versatile, capable of being used for cryoEM and/or as crystallization chaperones for X-ray crystallography. Although the cryoEM workflow described here produces modest resolution structures, it must be considered that these complexes are small by cryoEM standards at $35 \mathrm{kDa}$. We estimate that their already structurally detailed maps could be improved to 3-4 $\AA$ using $300 \mathrm{keV}$ microscopes (SI Appendix, Fig. S5). Achieving these resolutions would equal current crystallography results for claudin/cCpE complexes, and have further advantages like faster structural throughput timeframes and bypassing crystallization and diffraction bottlenecks. Thus, COPs could be employed as tools to rapidly expand structural knowledge of other claudin orthologs or paralogs that bind enterotoxins. In this and their potential obstructing capacities, COPs and COP-like sFabs may provide new insights useful for developing treatments for $\mathrm{CpE}$-based diseases or to aid design of novel $\mathrm{cCpE}-$ and $\mathrm{CpE}-$ based therapeutics that modulate TJ barriers.

\section{METHODS}

Claudin-4 and Enterotoxin Expression and Purification. Methods followed those described previously $(\underline{22}, \underline{23})$. Briefly, claudin-4, cCpE, and $\mathrm{CpE}$ with $\mathrm{C}$-terminal decahistidine tags preceded by thrombin cleavage sites (claudin-4-His $10, \mathrm{cCpE}-\mathrm{His}_{10}$, and $\mathrm{CpE}-\mathrm{His}_{10}$ ) were cloned into pFastBac1 (ThermoFisher) and expressed in Tn5 (Trichoplusia ni, High Five, Expression Systems, LLC). Cell pellets resuspended in Lysis buffer (50 mM Tris pH 8.0, $150 \mathrm{mM} \mathrm{NaCl}, 1 \mathrm{mM}$ PMSF, and EDTA-free SigmaFast protease tablets (Sigma)) were sonicated, supplemented with $1 \mathrm{M} \mathrm{NaCl}$, then ultracentrifugation at 100,000 xg for 1 hour. For enterotoxins, the supernatant was saved, $15 \mathrm{mM}$ imidazole was added along with NiNTA resin, and the solution was incubated for 12 hours at $4^{\circ} \mathrm{C}$. For claudin- 4 , the supernatant was removed, and the membrane pellet was resuspended in Lysis buffer and the protein was solubilized with $1 \%(\mathrm{w} / \mathrm{v}) \mathrm{n}$-dodecyl- $\beta$-Dmaltopyranoside (DDM, Anatrace) and $0.04 \%$ cholesteryl hemisuccinate (CHS, Anatrace) overnight at $4^{\circ} \mathrm{C}$. Insoluble protein was removed by ultracentrifugation at $100,000 \mathrm{xg}$ for 30 minutes, and the supernatant was treated with $15 \mathrm{mM}$ imidazole along with NiNTA resin, and the solution was incubated for 12 hours at $4^{\circ} \mathrm{C}$. For claudin- 4 , the bound protein was captured and washed with 5 column volumes of Buffer A (50 $\mathrm{mM}$ Tris $\mathrm{pH}$ 7.4, $500 \mathrm{mM} \mathrm{NaCl}, 25 \mathrm{mM}$ imidazole, and 0.087\% DDM) and B (Buffer A containing 300 $\mathrm{mM} \mathrm{NaCl}$ and $40 \mathrm{mM}$ imidazole). Buffer $\mathrm{T}$ (50 mM Tris $\mathrm{pH} 8.0,150 \mathrm{mM} \mathrm{NaCl}$ and $0.04 \% \mathrm{DDM}$ ) was used to release and capture proteins from the resin after treatment with thrombin. For enterotoxins, purification was similar to claudin-4 except DDM was not added to buffers. These proteins were then used for sFab panning or biochemical, biophysical, and structural analyses. For enterotoxins used for binding studies, the proteins were eluted off of NiNTA using Elution buffer (Buffer T containing $400 \mathrm{mM}$ imidazole) to keep the His 10 tag. Eluted enterotoxin-His 10 was dialyzed in size-exclusion chromatography (SEC) buffer (10 mM Hepes $\mathrm{pH} 7.4,100 \mathrm{mM} \mathrm{NaCl}$, and 4\% glycerol), concentrated to $1 \mathrm{mg} / \mathrm{mL}$, then flashfrozen in liquid nitrogen and stored at $-80^{\circ} \mathrm{C}$ until use.

Generation and Validation of COPs Using Phage Display. DDM-solubilized claudin-4 was biotinylated using N-hydroxysuccinimide polyethylene glycol biotin (NHS-PEG4-biotin, ThermoFisher) by mixing 5.6 $\mu \mathrm{M}$ claudin-4 with $16.8 \mu \mathrm{M}$ NHS-PEG4-biotin, followed by incubation on ice for 2 hours. Excess cCpE with the His ${ }_{10}$ removed was added at a ratio of 1:1.5 (moles:moles), then free biotin and unbound cCpE was removed by loading the sample onto a Superdex 200 Increase 10/300 GL (Cytiva) equilibrated in SEC buffer containing $0.04 \%$ DDM. The sFab panning was performed using the sFab library E $(\underline{33}, \underline{34})$. Binding was assayed in Selection buffer ( $25 \mathrm{mM}$ Hepes $\mathrm{pH} 7.4,150 \mathrm{mM} \mathrm{NaCl}$, and $1 \%$ bovine serum albumin). A first round of panning was performed manually using $200 \mathrm{nM}$ of biotinylated claudin-4/cCpE in DDM immobilized onto magnetic beads, and following three washes with Selection buffer, the beads enriched for phage expressing claudin-4/cCpE-specific sFabs were used to infect log-phase $E$. coli XL1-Blue cells. Phages were amplified overnight in 2xYT media supplemented with $100 \mu \mathrm{g} / \mathrm{ml}$ ampicillin and M13-KO7 helper phage $\left(10^{9} \mathrm{pfu} / \mathrm{ml}\right)$. Selection stringency was then increased by four additional rounds of panning 
using decreasing claudin-4/cCpE concentrations down to $\sim 20 \mathrm{nM}$. For each round, the amplified phage pool from each preceding round was used as the input. For rounds two to five, panning was performed semiautomatically using a Kingfisher magnetic beads handler (ThermoFisher). Non-specific binding of sFabs to detergent was reduced by using $>0.87 \%$ DDM in later rounds. Bound phage particles were removed by elution from beads using 1\% Fos-choline- 12.

The initial validation was performed by single-point phage ELISA using individual clones from later selection rounds. ELISAs were performed in 96-well plates (Nunc) coated with $2 \mu \mathrm{g} / \mathrm{ml}$ neutravidin and blocked with Selection buffer. E. coli XL1-Blue colonies containing phagemids were used to inoculate 400 $\mu 12 \mathrm{xYT}$ media containing $100 \mu \mathrm{g} / \mathrm{ml}$ ampicillin and $10^{9} \mathrm{pfu} / \mathrm{ml} \mathrm{M} 13-\mathrm{KO} 7$ helper phage. Phages were amplified overnight in 96-well deep-well blocks at $37^{\circ} \mathrm{C}$ with shaking at $280 \mathrm{rpm}$. Phages were then diluted 1:10 into Selection buffer and assayed against claudin-4/cCpE in DDM or buffer containing only DDM micelles. Biotinylated claudin- $4 / \mathrm{cCpE}$ was immobilized at room temperature for $30 \mathrm{~min}$ then incubated with phage dilutions. Bound phage were detected with TMB substrate (ThermoFisher) following a 30 minutes incubation with horseradish peroxidase-conjugated anti-M13 monoclonal antibody (GE Healthcare). Absorbance was measured at $450 \mathrm{~nm}$ after quenching the reaction with $1.0 \mathrm{M} \mathrm{HCl}$. Wells containing 1\% DDM were used to detect non-specific binding.

COP Expression and Purification. Two sFabs termed $\underline{\mathrm{CpE}}$ Obstructing Protein-2 and -3 (COPs), from phage ELISA were selected and sequenced at the University of Chicago Comprehensive Cancer Center DNA Sequencing facility. Unique clones for each COP were sub-cloned in pRH2.2 using the In-Fusion Cloning kit (Takara Bio). Sequence-verified COPs were transformed into E. coli BL21-Gold cells (Agilent) and then used to inoculate overnight cultures. The inoculates were then used to seed $1 \mathrm{~L}$ of $2 \times \mathrm{XT}$ media containing $100 \mu \mathrm{g} / \mathrm{ml}$ ampicillin. Cultures were grown to an $\mathrm{OD}_{600}$ of 0.8 , induced for 4 hours at $37^{\circ} \mathrm{C}$, then cells were harvested using centrifugation. Cell pellets were resuspended in COP Lysis buffer (20 mM Hepes $\mathrm{pH} 7.4,150 \mathrm{mM} \mathrm{NaCl}, 0.5 \mathrm{mM} \mathrm{MgCl} 2,1 \mathrm{mM}$ PMSF, and $1 \mu \mathrm{g} / \mathrm{ml}$ DNase I). Cells were sonicated and lysates were incubated at $60^{\circ} \mathrm{C}$ for 30 minutes to remove proteolyzed fragments. Samples were cooled rapidly on ice then cleared by centrifugation. Supernatants were filtered by $0.45 \mu \mathrm{m}$ and loaded onto a 5 mL HiTrap MabSelect SuRe column (GE Healthcare) equilibrated with COP Wash buffer (20 mM Hepes pH 7.4 and $500 \mathrm{mM} \mathrm{NaCl}$ ). The column was washed with 10 column volumes of COP Wash buffer and COPs were eluted with $0.1 \mathrm{M}$ acetic acid. Eluted COPs were loaded onto a $1 \mathrm{~mL}$ Resource S column (GE Healthcare) equilibrated with COP Buffer A $(50 \mathrm{mM}$ sodium acetate $\mathrm{pH} 5.0)$ and washed with 10 column volumes of this buffer. The COPs were eluted by linear $0-50 \%$ gradient with COP Buffer B (COP Buffer A containing $2 \mathrm{M} \mathrm{NaCl}$ ). COP-containing fractions were pooled and dialyzed overnight at $4{ }^{\circ} \mathrm{C}$ in $\mathrm{SEC}$ buffer.

Biochemical Characterization of COP Binding. We used post-NiNTA purified un-tagged claudin-4 and enterotoxins, and post-affinity purified COPs for these analyses. For claudin-4/COP and enterotoxin/COP studies, $50 \mu \mathrm{g}$ enterotoxins was used and excess COPs were added at a 1:1.2 molar ratio, incubated at room temperature for 1 hour, concentrated, $0.2 \mu \mathrm{m}$ filtered, then loaded onto a Superdex 200 column equilibrated in SEC buffer containing 0.04\% DDM. For claudin-4/enterotoxin/COP studies, $50 \mu \mathrm{g}$ claudin-4 was used and excess enterotoxins were added at a 1:1.2 molar ratio, then COPs were added at a 1:1 molar ratio to enterotoxins. Complexes were incubated, concentrated and filtered, and loaded onto a Superdex 200 column equilibrated in SEC buffer and 0.04\% DDM. Complex formation was assessed by observed decreases in the elution times of the uncomplexed peak fractions. Peak fractions containing complexes were pooled, unboiled, and evaluated for the presence of each protein by SDS-PAGE using 4-20\% agarose gradient gels.

Biophysical Characterization of COP Binding Using Bio-layer Interferometry (BLI). BLI analyses were performed at $25^{\circ} \mathrm{C}$ at an acquisition rate of $5 \mathrm{~Hz}$ averaged by 20 using an OctetC BLItz System (FortéBio/Sartorius) with assays were designed and setup using Blitz Pro 1.3 Software. A typical experiment consisted of the following steps: sensor equilibration (30 seconds), protein loading (200 
seconds), baseline (60 seconds), and association and dissociation (300 seconds each). For the loading step, $4 \mu 1$ of proteins were loaded in the drop holder, while all other steps were performed in transparent $600 \mu 1$ microtubes using $250 \mu \mathrm{l}$ sample volumes. All measurements were performed in SEC buffer containing $0.04 \%$ DDM. For enterotoxin/COP studies, $5 \mu \mathrm{M}(70 \mu \mathrm{g} / \mathrm{ml})$ of wild type or mutant cCpE-His 10 or CpEHis $_{10}$ were loaded on NiNTA (Dip and $\operatorname{Read}^{\mathrm{TM}}$ ) sensors then dipped into a $0-1 \mu \mathrm{M}$ range of four concentrations of COPs for the association steps. For claudin-4/enterotoxin/COP studies, $5 \mu \mathrm{M}$ DDMsolubilized claudin-4 biotinylated with NHS-PEG4-biotin as before was pre-complexed with excess wild type or mutant enterotoxins without a $\mathrm{His}_{10}$ at a ratio of 1:2 (moles:moles) for 1 hour at room temperature. Claudin-4/enterotoxin complexes were loaded on Streptavidin-SA (Dip and Read ${ }^{\mathrm{TM}}$ ) sensors and the measurements were performed as above using $0-10 \mu \mathrm{M}$ range of concentrations depending on the analyte. For all studies, measurements were repeated in at least duplicate, and the time courses for association and dissociation were fit to one-site binding model using the BLItz Pro 1.3 Software. No significant nonspecific binding of COPs to unloaded NiNTA or streptavidin sensors were detected.

CryoEM Sample Preparation. DDM-solubilized claudin-4 was exchanged into 2,2-didecylpropane-1,3bis- $\beta$-D-maltopyranoside (LMNG, Anatrace) or amphipol A8-35 (Anatrace). For exchange into LMNG the protein bound to NiNTA resin was sequentially washed with Buffer A, B, and T containing 0.087, 0.087, and $0.04 \% \mathrm{LMNG}$, respectively, before releasing the bound protein from resin via thrombin cleavage. To prepare sample in amphipol A8-35, $1 \mathrm{mg}$ of post-thrombin digested claudin-4 in DDM was treated with 4 mg of amphipol $(1: 4 \mathrm{w} / \mathrm{w})$ for 2 hours before removing detergent via addition of $400 \mathrm{mg}$ SM-2 biobeads (Bio-Rad). Excess cCpE was added to each claudin-4 sample at a molar ratio of 1:1.5, incubated at room temperature for 1 hour, then excess COPs were added at a 1.5:1 ratio to $\mathrm{cCpE}$. After 1 hour at room temperature each sample was concentrated, $0.2 \mu \mathrm{m}$ filtered, then loaded onto a Superdex 200 Increase $10 / 300$ GL equilibrated in SEC buffer without glycerol but with $0.003 \%$ LMNG or no detergent for amphipol samples. Peak fractions from SEC containing claudin-4/cCpE/COP complexes were collected and concentrated to $6-8 \mathrm{mg} / \mathrm{mL}$ for use in cryoEM analyses.

Grids for cryo-EM analyses were prepared using a Vitrobot Mark IV (ThermoFisher) plunge freezing apparatus. Aliquots $(3 \mu \mathrm{L})$ of claudin-4/cCpE/COP complexes in LMNG or amphipol were applied to Quantifoil R1.2/1.3200 mesh grids that were glow-discharged for 45 seconds at $15 \mathrm{~mA}$ in a Pelco easiGlow (Ted Pella Inc) instrument. Protein solutions were applied to grids at $4^{\circ} \mathrm{C}$ and $100 \%$ relative humidity and allowed to adsorb on the grid for $\sim 30$ seconds before blotting. Grids were blotted for 5-8 seconds with a blot force of 1 , and plunge frozen into liquid ethane cooled by liquid nitrogen. Grids were stored in liquid nitrogen prior to imaging.

CryoEM Data Collection and Processing. CryoEM data collection was performed on a Talos Arctica (ThermoFisher) equipped with a Falcon III (ThermoFisher) direct electron detector at Michigan State University. Grids were screened for thin ice and good particle distribution, and data collection was performed using EPU software (ThermoFisher). Movies of claudin-4/cCpE/COP-2 in LMNG were collected on the Falcon III detector in counting mode at 92,000x magnification with a pixel size of $1.12 \AA$, a defocus range of 0.8 to $2.6 \mu \mathrm{m}$, and a total dose of $\sim 32$ electron $/ \AA^{2}$ fractionated over 51 total frames. Movies of claudin-4/cCpE/COP-3 in amphipol were collected at 120,000x magnification with a pixel size of $0.87 \AA$, a defocus range of -1 to $-2.2 \mu \mathrm{m}$, and a total dose of $\sim 40$ electron $/ \AA^{2}$ fractionated over 42 total frames.

All micrograph and particle processing was performed in CryoSPARC (40). Patch-motion correction and patch-CTF correction were used to correct for beam-induced motion and calculate CTF parameters from the motion-corrected micrographs. Blob-based template picking followed by $2 \mathrm{D}$ classification was used to generate templates that were subsequently used for template-based particle picking. Particles identified from this template-based picking procedure were subjected to several rounds of $2 \mathrm{D}$ classification, followed by ab initio 3D reconstruction, heterogeneous refinement, and non-uniform refinement. In the case of the 
COP-3 complex, local refinement using a soft mask around $\mathrm{cCpE}$ and COP-3 was also used to improve resolution at the complex's interface.

CryoEM Model Building, Refinement, and Structure Determination. Using post-processing maps we first built the structure of claudin-4/cCpE/COP-2 using the $6.9 \AA$ map and the crystal structure of claudin4 in complex with cCpE (PDB ID 7KP4) (23). 7PK4 was manually docked by placing the four TMs of claudin-4 into density present in the LMNG micelle using Coot (41). TM placement was validated using density corresponding to bulky side chains, like Trp18 in TM1 of claudin-4. Initial placement of the TM region showed that only minor alterations to claudin-4's ECS and cCpE would be required to fit well within the cryoEM map. For this part of the structure, the map volume allowed placement of most claudin-4 and $\mathrm{cCpE}$ residues, with the exception of claudin-4's C-terminus and cCpE's N-terminus. We next built COP2 by first using a high-resolution crystal structure of a sFab (PDB ID 6CBV). COP-2 was manually docked into the additional cryoEM density using Coot, and then divergent residues of COP-2 were manually mutating from the $6 \mathrm{CBV}$ template using sequence alignments as a guide (느). Once the three proteins were fit manually, each of the four protein chains were rigid body refined in Coot to place the structure within the cryoEM map volume. Model building was done using Coot and once complete, the structure was further refined using a combination of molecular dynamics flexible fitting simulations using Namdinator followed by real-space refinement of the model into the cryoEM density map using Phenix phenix.real_space_refine $(43,44)$. The final structural model required secondary structure and Ramachandran restraints to optimize the model-to-map fit and overall geometry. SI Appendix, Table S1 shows data collection, refinement, and validation statistics for the claudin-4/cCpE/COP-2 structure.

The structure of claudin-4/cCpE/COP-3 was determined using both a masked and unmasked strategy, which produced focused and whole maps that resolved to 3.8 and 5.0 $\AA$, respectively. A mask was applied to COP3 in the complex in the former case while all three proteins were included in the latter. The final model of the claudin-4/cCpE/COP-2 complex was manually docked then COP-3 was made from COP-2 by mutating divergent residues using sequence alignments. Each protein chain in the complex was then individually refined as rigid bodies into the cryoEM maps using Coot. While the focused map had added features for COP-3 it lacked features for claudin- 4 and $\mathrm{cCpE}$ such that refinements resulted in poor model-to-map fits. Thus, all three proteins were built and fit manually within the whole map volume using Coot and the final model was refined using Namdinator and Phenix phenix.real_space_refine using secondary structure and Ramachandran restraints $(\underline{43}, \underline{44})$. SI Appendix, Table S1 shows data collection, refinement, and validation statistics for the claudin-4/cCpE/COP-3 structure.

The programs used to visualize and build the structures included Coot, PyMOL, and Chimera, refined using Phenix, and figures were made using PyMOL—using the SBGrid Consortium Software Suite (1ㅗ, 45-48).

Mutagenesis of $\mathbf{c C p E}$. The pFastBac1 cloned $\mathrm{cCpE}^{\mathrm{H}} \mathrm{His} \mathrm{s}_{10}$ was altered using site-directed mutagenesis. Mutants were generated with the following forward primers and their equivalent reverse compliments: $\mathrm{cCpE}^{1} 5^{\prime}$-gcgcggatccgccaccgcatcaacggacattatgaaagaaatcctcgac-3';

$\mathrm{cCpE}^{2} 5^{\prime}$-ccatgtcaacggacattgaaaaagaagccgccgcttagctgctgcaacagaacgc-3';

$\mathrm{cCpE}^{2 \mathrm{~L}} 5^{\prime}$-ccatgtcaacggacattgaaaaagaagccgecgecgecgetgctgcaacagaacgc-3';

$\mathrm{cCpE}^{3} 5^{\prime}$ '-gttgactttaacatttactccaacgccgecgctgccettgtcaaactcgaacaatcgctc-3';

$\mathrm{cCpE}^{4}$ 5'-catttactccaacaacttcaataacgctgccaaagccgcacaatcgetcggagatggtg-3'. Protocols for expression and purification of all cCpE-His 10 mutants were identical to those of wild type $\mathrm{cCpE}-\mathrm{His}_{10}$.

Structural Modeling of Claudin-bound CpE Pore. A model of claudin-4/CpE was made by superposing $\mathrm{CpE}$ (PDB ID 3AM2) onto cCpE from our cryoEM structure of the COP-2 complex, making a claudin$4 / \mathrm{CpE} / \mathrm{COP}$ model. To approximate the $\mathrm{CpE} \beta$-pore, we modeled it after lysenin, a $\beta$-pore toxin with homology to $\mathrm{CpE}$, using an available cryoEM structure (PDB ID 5GAQ) $(37,49)$. Using a crystal structure of the lysenin monomer (PDB ID 3ZXD), we superposed 3ZXD onto one monomer of the 5GAQ nonamer, 
then superposed the $\mathrm{CpE}$ portion of our claudin-4/CpE/COP model onto 3ZXD (50). We next removed $3 Z X D$. This process integrated claudin-4/CpE/COP into lysenin, making the model for the claudin-4-bound $\mathrm{CpE} \beta$-pore. These models were used to predict COP influence on $\mathrm{CpE}$-induced cytotoxicity.

Cell-Based Cytotoxicity Assay. Recombinant baculoviruses containing claudin-4-His ${ }_{10}$ were produced using established methods and the cytotoxicity assay was performed using methods previously described (23). To 18 wells of a 24-well cell culture plate, $0.5 \mathrm{~mL}$ of $1.0 \times 10^{6}$ adherent $\mathrm{Sf9}$ (Spodoptera frugiperda, Expression Systems) cells were added. After 1 hour at $27^{\circ} \mathrm{C}$, virus containing claudin-4-His 10 was added at a MOI of 1.0. Cultures were rocked gently then placed at $27^{\circ} \mathrm{C}$ for 48 hours. The 18 wells were divided into three, 6 -well groups. To duplicate wells of group one and three, $22 \mu \mathrm{g}(0.9 \mu \mathrm{M})$ of COP-2 was added; to a second pair of wells, $22 \mu \mathrm{g}(0.9 \mu \mathrm{M})$ of COP-3 was added; and to the third pair of wells no COP was added. To duplicate wells of group two, $100 \mu \mathrm{g}(4 \mu \mathrm{M})$ of COP-2 was added; to a second pair of wells, 100 $\mu \mathrm{g}(4 \mu \mathrm{M})$ of COP-3 was added; and to the third pair of wells no COP was added. For group one, nothing more was added; this group contained only cells expressing claudin-4-His ${ }_{10}$. For group two, $25 \mu \mathrm{g}(3.3 \mu \mathrm{M})$ purified $\mathrm{cCpE}$ in SEC buffer was added to the culture medium. For group three, $12.5 \mu \mathrm{g}(0.7 \mu \mathrm{M}) \mathrm{CpE}$ in SEC buffer was added to the culture medium. The COP amounts added correspond to 1.2-fold molar excess of COP to enterotoxin. After addition of COPs and/or enterotoxins, $\mathrm{Sf} 9$ cells were placed at $27^{\circ} \mathrm{C}$ for 12 hours, then $\mathrm{CpE}$ cytotoxicity was quantified using a cell viability analysis. This was done by gently removing $250 \mu \mathrm{L}$ of Sf9 cells from each well, centrifuging at $200 \mathrm{xg}$ for 2 minutes, removing $200 \mu \mathrm{L}$ of media, then adding $50 \mu \mathrm{L}$ of $0.04 \%$ trypan blue. After 5 minutes, $10 \mu \mathrm{L}$ of stained cells were transferred to a Countess cell counting chamber slides and counted automatically using a Countess 3 automated cell counter (ThermoFisher). Each well was counted two times independently (representing four readings per sample, 36 for nine samples). Viability counts consisted of dividing the total number of live cells (unstained) by the total number of cells. Average cell viability for enterotoxin treated cells were compared to Sf9 cells expressing claudin-4 that were not treated with either enterotoxins or COPs. Data was plotted as a mean with standard deviation in GraphPad Prism 9 for macOS.

\section{ACKNOWLEDGMENTS}

Research reported in this publication was supported by the National Institute of General Medical Sciences of the National Institutes of Health under Award Number R35GM138368 (to A.J.V.) and R01GM117372 (to A.A.K). The content is solely the responsibility of the authors and does not necessarily represent the official views of the National Institutes of Health. Also, we are grateful to the RTSF Cryo-Electron Microscopy Core at Michigan State University and Dr. Sundarraman Subramanian for providing time and support for cryoEM data collection.

\section{AUTHOR CONTRIBUTIONS}

A.J.V. designed the research; performed claudin and enterotoxin expression, purification and sample preparation for structure/function studies, biochemical characterization, cytotoxicity assays; determined cryoEM structures; analyzed data; made figures; and wrote the paper; B.J.O. performed the cryoEM, determined cryoEM structures, and edited the paper; P.K.D. performed sFab phage display screening and validation; P.K.D. and S.K.E. performed COP expression and purification; S.R. performed binding measurements; S.R. and C.O. performed claudin and enterotoxin expression and purification for binding studies; A.A.K. developed the sFab phage display library, designed the sFab-related research, and edited the paper.

\section{COMPETING INTERESTS}

The authors declare no competing interests.

\section{DATA AVAILABILITY}

The cryoEM structure of claudin-4/cCpE/COP-2 has accession code 7DTM in the Protein Data Bank (PDB) and cryoEM maps of this complex have been deposited to the Electron Microscopy Data Bank under 
accession code EMD-25834. The cryoEM structure of claudin-4/cCpE/COP-3 has PDB accession code 7DTM and cryoEM maps of this complex have been deposited to the Electron Microscopy Data Bank under accession code EMD-25835 (whole) and EMD-25836 (focused).

\section{REFERENCES}

1. Anderson JM \& Van Itallie CM (2009) Physiology and function of the tight junction. Cold Spring Harb Perspect Biol 1:a002584.

2. Chiba H, Osanai M, Murata M, Kojima T, \& Sawada N (2008) Transmembrane proteins of tight junctions. Biochim Biophys Acta 1778:588-600.

3. Furuse M, Fujita K, Hiiragi T, Fujimoto K, \& Tsukita S (1998) Claudin-1 and -2: novel integral membrane proteins localizing at tight junctions with no sequence similarity to occludin. J Cell Biol 141:1539-1550.

4. Katahira J, et al. (1997) Clostridium perfringens enterotoxin utilizes two structurally related membrane proteins as functional receptors in vivo. J Biol Chem 272:26652-26658.

5. McClane BA (2001) The complex interactions between Clostridium perfringens enterotoxin and epithelial tight junctions. Toxicon 39:1781-1791.

6. Rood JI, et al. (2018) Expansion of the Clostridium perfringens toxin-based typing scheme. Anaerobe 53:5-10.

7. Freedman JC, Shrestha A, \& McClane BA (2016) Clostridium perfringens Enterotoxin: Action, Genetics, and Translational Applications. Toxins (Basel) 8.

8. Uzal FA, et al. (2014) Towards an understanding of the role of Clostridium perfringens toxins in human and animal disease. Future Microbiol 9:361-377.

9. Hoffmann S, Batz MB, \& Morris JG, Jr. (2012) Annual cost of illness and quality-adjusted life year losses in the United States due to 14 foodborne pathogens. J Food Prot 75:1292-1302.

10. Scallan E, et al. (2011) Foodborne illness acquired in the United States--major pathogens. Emerg Infect Dis 17:7-15.

11. Kirk MD, et al. (2015) World Health Organization Estimates of the Global and Regional Disease Burden of 22 Foodborne Bacterial, Protozoal, and Viral Diseases, 2010: A Data Synthesis. PLoS Med 12:e1001921.

12. Scharff RL (2012) Economic burden from health losses due to foodborne illness in the United States. J Food Prot 75:123-131.

13. Suzuki H, Hosomi K, Nasu A, Kondoh M, \& Kunisawa J (2018) Development of Adjuvant-Free Bivalent Food Poisoning Vaccine by Augmenting the Antigenicity of Clostridium perfringens Enterotoxin. Front Immunol 9:2320.

14. Duncan CL, Strong DH, \& Sebald M (1972) Sporulation and enterotoxin production by mutants of Clostridium perfringens. J Bacteriol 110:378-391.

15. Veshnyakova A, et al. (2010) On the interaction of Clostridium perfringens enterotoxin with claudins. Toxins (Basel) 2:1336-1356.

16. Vecchio AJ, Rathnayake SS, \& Stroud RM (2021) Structural basis for Clostridium perfringens enterotoxin targeting of claudins at tight junctions in mammalian gut. Proc Natl Acad Sci US A 118.

17. Robertson SL, et al. (2007) Compositional and stoichiometric analysis of Clostridium perfringens enterotoxin complexes in Caco-2 cells and claudin 4 fibroblast transfectants. Cell Microbiol 9:2734-2755.

18. Mitchell LA \& Koval M (2010) Specificity of interaction between clostridium perfringens enterotoxin and claudin-family tight junction proteins. Toxins (Basel) 2:1595-1611.

19. Saitoh Y, et al. (2015) Structural insight into tight junction disassembly by Clostridium perfringens enterotoxin. Science 347:775-778.

20. Shinoda T, et al. (2016) Structural basis for disruption of claudin assembly in tight junctions by an enterotoxin. Sci Rep 6:33632. 
21. Nakamura S, et al. (2019) Morphologic determinant of tight junctions revealed by claudin-3 structures. Nat Commun 10:816.

22. Vecchio AJ \& Stroud RM (2019) Claudin-9 structures reveal mechanism for toxin-induced gut barrier breakdown. Proc Natl Acad Sci U S A 116:17817-17824.

23. Vecchio AJ, Rathnayake, S.S., Stroud, R.M. (2021) Structural basis for Clostridium perfringens enterotoxin targeting of claudins at tight junctions in mammalian gut. Proc Natl Acad Sci US A 118.

24. Maeda T, et al. (2012) Claudin-4-targeted therapy using Clostridium perfringens enterotoxin for prostate cancer. Prostate 72:351-360.

25. Pahle J, et al. (2017) Rapid eradication of colon carcinoma by Clostridium perfringens Enterotoxin suicidal gene therapy. BMC Cancer 17:129.

26. Tachibana K, et al. (2020) Tight Junction Modulating Bioprobes for Drug Delivery System to the Brain: A Review. Pharmaceutics 12.

27. Torres JB, et al. (2020) Radiolabeled cCPE Peptides for SPECT Imaging of Claudin-4 Overexpression in Pancreatic Cancer. J Nucl Med 61:1756-1763.

28. Bukowska MA \& Grutter MG (2013) New concepts and aids to facilitate crystallization. Curr Opin Struct Biol 23:409-416.

29. Dominik PK, et al. (2016) Conformational Chaperones for Structural Studies of Membrane Proteins Using Antibody Phage Display with Nanodiscs. Structure 24:300-309.

30. $\quad \mathrm{Wu} \mathrm{S}$, et al. (2012) Fabs enable single particle cryoEM studies of small proteins. Structure 20:582592.

31. Dutka P, et al. (2019) Development of "Plug and Play" Fiducial Marks for Structural Studies of GPCR Signaling Complexes by Single-Particle Cryo-EM. Structure 27:1862-1874 e1867.

32. Bloch JS, et al. (2021) Development of a universal nanobody-binding Fab module for fiducialassisted cryo-EM studies of membrane proteins. Proc Natl Acad Sci U S A 118.

33. Fellouse FA, Wiesmann C, \& Sidhu SS (2004) Synthetic antibodies from a four-amino-acid code: a dominant role for tyrosine in antigen recognition. Proc Natl Acad Sci U S A 101:12467-12472.

34. Fellouse FA, et al. (2007) High-throughput generation of synthetic antibodies from highly functional minimalist phage-displayed libraries. J Mol Biol 373:924-940.

35. Krissinel E \& Henrick K (2007) Inference of macromolecular assemblies from crystalline state. $J$ Mol Biol 372:774-797.

36. Shinoda T, et al. (2016) Cell-free methods to produce structurally intact mammalian membrane proteins. Sci Rep 6:30442.

37. Cirauqui N, Abriata LA, van der Goot FG, \& Dal Peraro M (2017) Structural, physicochemical and dynamic features conserved within the aerolysin pore-forming toxin family. Sci Rep 7:13932.

38. Mitic LL, Unger VM, \& Anderson JM (2003) Expression, solubilization, and biochemical characterization of the tight junction transmembrane protein claudin-4. Protein Sci 12:218-227.

39. Suzuki H, et al. (2014) Crystal structure of a claudin provides insight into the architecture of tight junctions. Science 344:304-307.

40. Punjani A, Rubinstein JL, Fleet DJ, \& Brubaker MA (2017) cryoSPARC: algorithms for rapid unsupervised cryo-EM structure determination. Nat Methods 14:290-296.

41. Emsley P, Lohkamp B, Scott WG, \& Cowtan K (2010) Features and development of Coot. Acta Crystallogr D Biol Crystallogr 66:486-501.

42. Mukherjee S, et al. (2020) Synthetic antibodies against BRIL as universal fiducial marks for singleparticle cryoEM structure determination of membrane proteins. Nat Commun 11:1598.

43. Afonine PV, et al. (2018) Real-space refinement in PHENIX for cryo-EM and crystallography. Acta Crystallogr D Struct Biol 74:531-544.

44. Kidmose RT, et al. (2019) Namdinator - automatic molecular dynamics flexible fitting of structural models into cryo-EM and crystallography experimental maps. IUCrJ 6:526-531.

45. Schrödinger (The PyMOL Molecular Graphics System Version 2.2.3). 
46. Pettersen EF, et al. (2004) UCSF Chimera--a visualization system for exploratory research and analysis. J Comput Chem 25:1605-1612.

47. Adams PD, et al. (2010) PHENIX: a comprehensive Python-based system for macromolecular structure solution. Acta Crystallogr D Biol Crystallogr 66:213-221.

48. Morin A, et al. (2013) Collaboration gets the most out of software. Elife 2:e01456.

49. Bokori-Brown M, et al. (2016) Cryo-EM structure of lysenin pore elucidates membrane insertion by an aerolysin family protein. Nat Commun 7:11293.

50. De Colibus L, et al. (2012) Structures of lysenin reveal a shared evolutionary origin for poreforming proteins and its mode of sphingomyelin recognition. Structure 20:1498-1507.

51. Notredame C, Higgins DG, \& Heringa J (2000) T-Coffee: A novel method for fast and accurate multiple sequence alignment. J Mol Biol 302:205-217. 


\section{TABLES AND FIGURES}

Table 1: Affinities and Kinetics of COP Binding to Claudin-4/cCpE and Enterotoxins

\begin{tabular}{ccccc}
\hline COP & Complex/Protein & $\mathbf{k}_{\text {on }}(\mathbf{1} / \mathbf{M s})$ & $\mathbf{k}_{\text {off }}(\mathbf{1} / \mathbf{s})$ & $\mathbf{K}_{\mathbf{D}}(\mathbf{n M})$ \\
\hline \multirow{2}{*}{ COP-2 } & \multirow{2}{*}{ claudin-4/cCpE } & $1.5 \times 10^{4}$ & $7.8 \times 10^{-4}$ & $\mathbf{5 2 . 3}$ \\
& & $\pm 0.1 \times 10^{4}$ & $\pm 0.6 \times 10^{-4}$ & \pm 3.0 \\
& \multirow{2}{*}{$\mathrm{c} p \mathrm{pE}$} & $7.2 \times 10^{3}$ & $4.8 \times 10^{-4}$ & $\mathbf{6 7 . 6}$ \\
& & $\pm 0.6 \times 10^{3}$ & $\pm 0.4 \times 10^{-4}$ & \pm 11.7 \\
& \multirow{2}{*}{ claudin-4/CpE } & $1.5 \times 10^{2}$ & $1.2 \times 10^{-3}$ & $\mathbf{7 , 8 0 0 . 0}$ \\
& & $\pm 0.6 \times 10^{2}$ & $\pm 0.6 \times 10^{-3}$ & \pm 750.0 \\
\hline \multirow{2}{*}{ COP-3 } & \multirow{2}{*}{ claudin-4/cCpE } & $2.9 \times 10^{4}$ & $2.7 \times 10^{-3}$ & $\mathbf{9 8 . 4}$ \\
& & $\pm 0.7 \times 10^{4}$ & $\pm 0.5 \times 10^{-3}$ & \pm 11.0 \\
& \multirow{2}{*}{$\mathrm{cCpE}$} & $1.0 \times 10^{4}$ & $1.0 \times 10^{-3}$ & $\mathbf{1 3 7 . 6}$ \\
& & $\pm 0.1 \times 10^{4}$ & $\pm 0.1 \times 10^{-3}$ & \pm 6.2 \\
& \multirow{2}{*}{ claudin-4/CpE } & $1.2 \times 10^{3}$ & $10.8 \times 10^{-2}$ & $\mathbf{9 , 0 5 0 . 0}$ \\
& & $\pm 0.2 \times 10^{3}$ & $\pm 0.7 \times 10^{-2}$ & $\pm 1,300.0$ \\
\hline
\end{tabular}



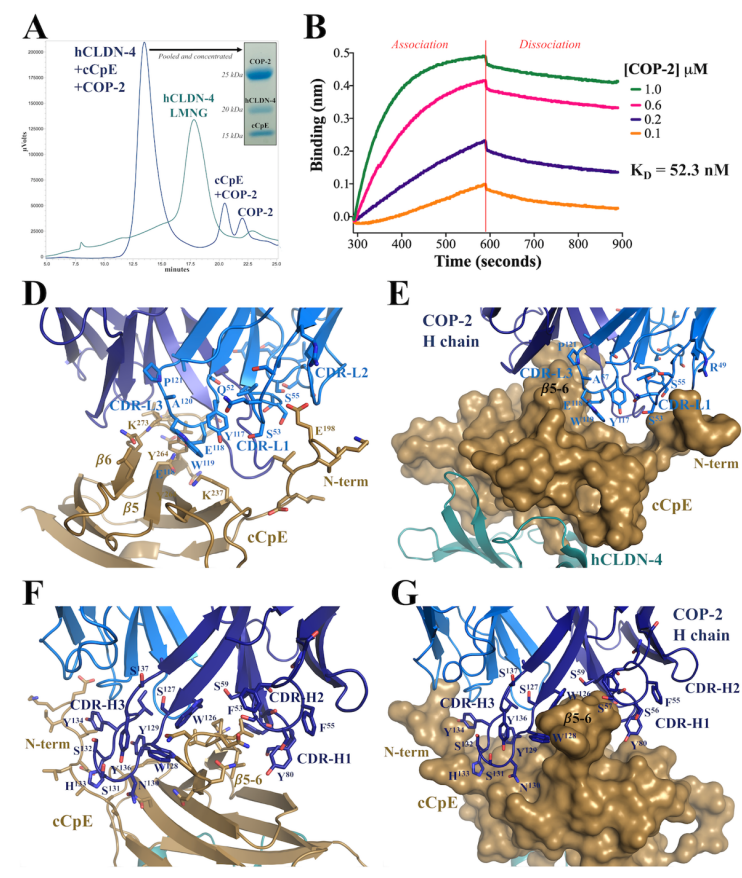

$\mathbf{E}$
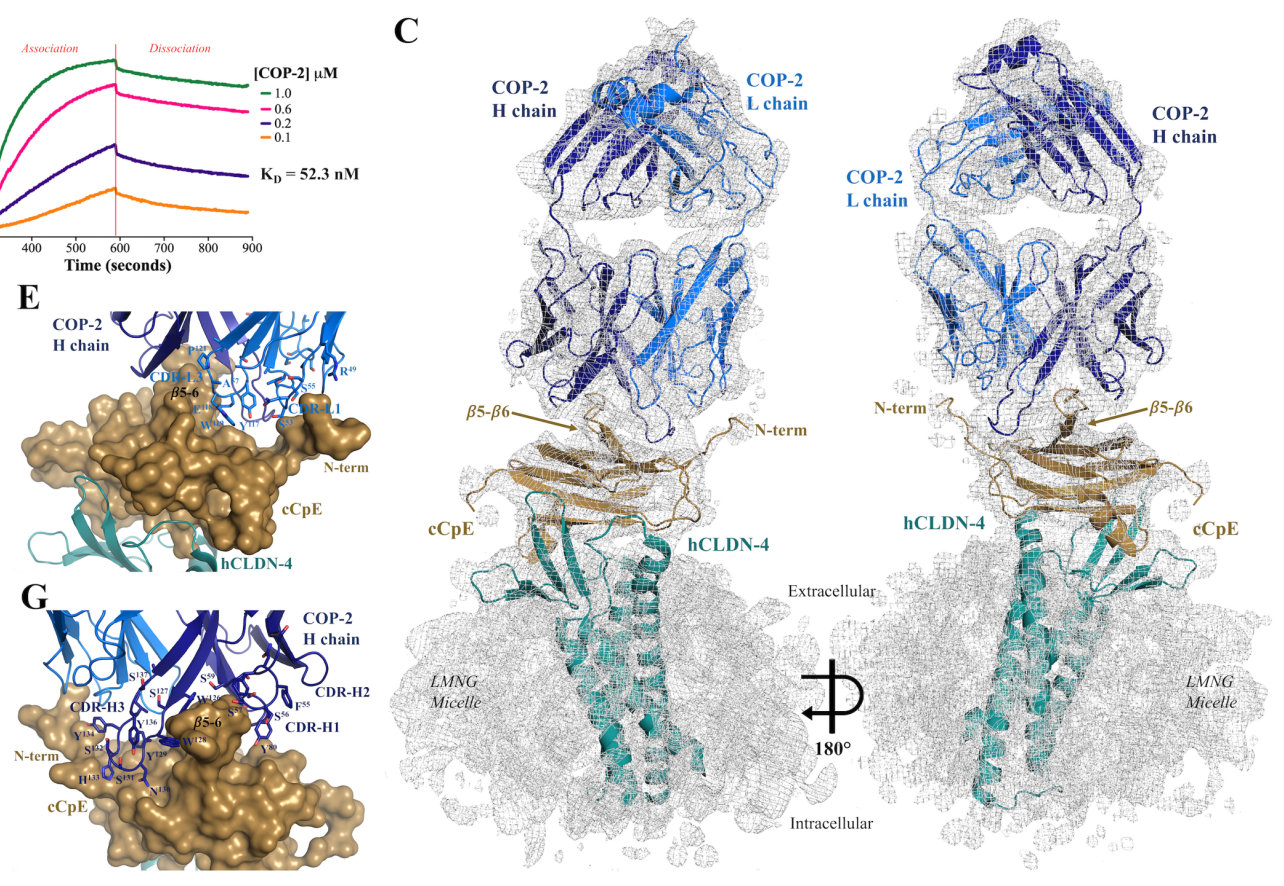

Figure 1. Structure and Function of COP-2 Binding to Claudin-4/cCpE Complexes. (A) SEC purification of human claudin-4 (hCLDN-4)/cCpE/COP-2 complexes in LMNG detergent. (B) Binding of COP-2 to claudin-4/cCpE using bio-layer interferometry. (C) CryoEM structure of COP-2 (blue) bound to $\mathrm{cCpE}$ (copper) and hCLDN-4 (teal). Proteins are shown as cartoons within the cryoEM map (grey), which is contoured to 4.0 б. (D-G) Potential interactions between COP-2 (blue) and cCpE (copper) for: (D and E) Chain L (light blue); and (F and $\mathbf{G}$ ) Chain H (dark blue). COP-2 and cCpE are both represented as cartoons (D and $\mathbf{F}$ ) or, COP-2 as a cartoon and $\mathrm{cCpE}$ as a surface $(\mathbf{E}$ and $\mathbf{G})$. 

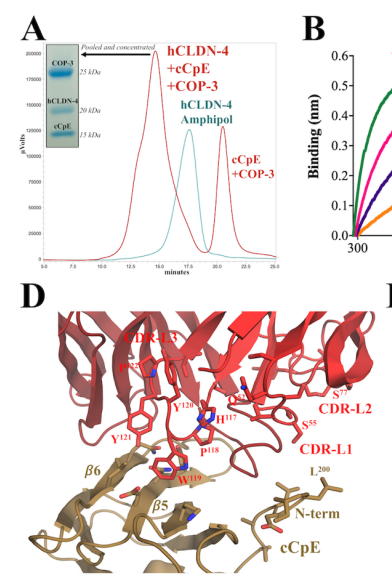

\section{F}

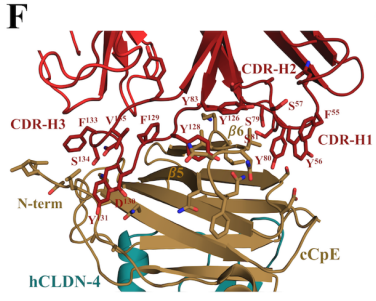

B

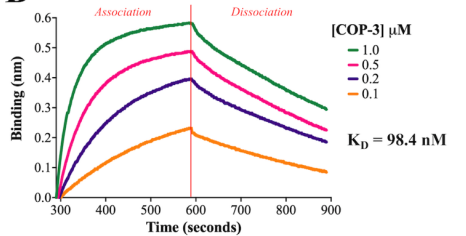

$\mathbf{E}$

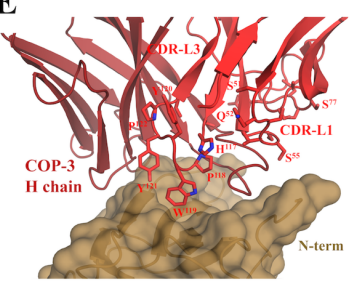

G

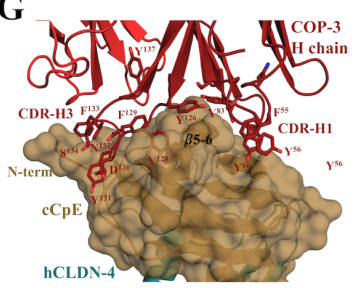

C

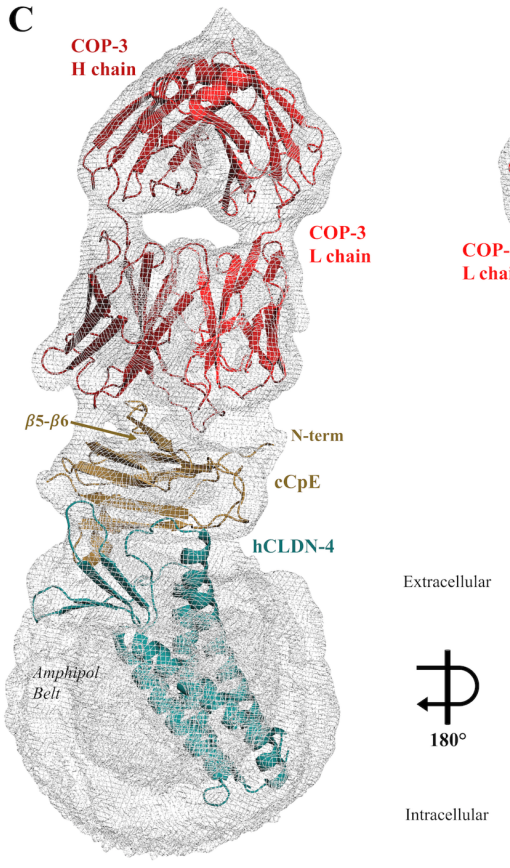

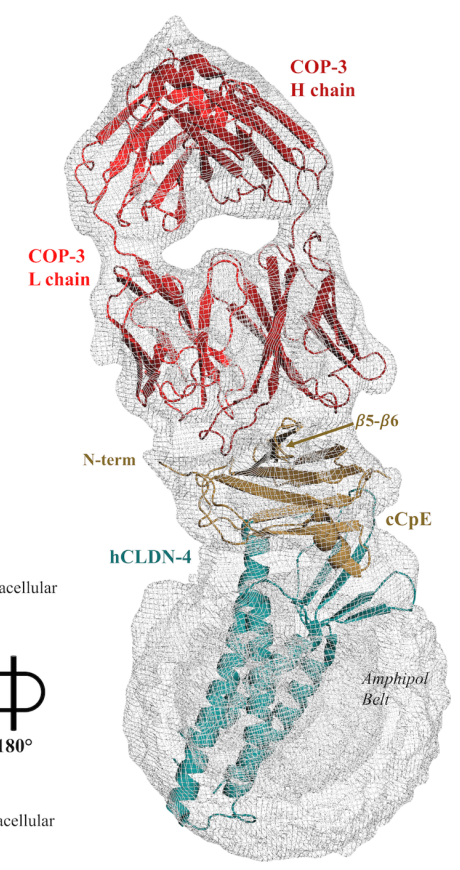

Figure 2. Structure and Function of COP-3 Binding to Claudin-4/cCpE Complexes. (A) SEC purification of human claudin-4 (hCLDN-4)/cCpE/COP-3 complexes in amphipol A8-35. (B) Binding of COP-3 to claudin-4/cCpE using bio-layer interferometry. (C) CryoEM structure of COP-3 (red) bound to $\mathrm{cCpE}$ (copper) and hCLDN-4 (teal). Proteins are shown as cartoons within the cryoEM map (grey), which is contoured to 5.0 б. (D-G) Potential protein/protein interactions between COP-3 (red) and cCpE (copper) for: (D and $\mathbf{E}$ ) Chain L (red); and (F and $\mathbf{G})$ Chain H (maroon). COP-3 and cCpE are both represented as cartoons (D and $\mathbf{F}$ ) or, COP-3 as a cartoon and $\mathrm{cCpE}$ as a surface (E and $\mathbf{G})$. 
Table 2: Mutant cCpE Sequences

\begin{tabular}{|c|c|c|}
\hline Mutant & cCpE $\mathbf{E}^{\text {mutant }}$ & Sequences \\
\hline \multirow{2}{*}{1} & $\mathrm{cCpE}^{\text {wildtype }}$ & ${ }^{192}$ STDIEKEILDLAAATERLN ${ }^{210}$ \\
\hline & $\mathrm{cCpE}^{\Delta 192-196}$ & ${ }^{197}$ KEILDLAAATERLN ${ }^{210}$ \\
\hline \multirow[b]{2}{*}{2} & $\mathrm{cCpE}^{\text {wildtype }}$ & ${ }^{192}$ STDIEKEILDLAAATERLN ${ }^{210}$ \\
\hline & $\mathrm{cCpE}^{\mathrm{Ala} 199-201}$ & ${ }^{192}$ STDIEKE $A A A$ LAAATERLN ${ }^{210}$ \\
\hline \multirow{2}{*}{$2 \mathrm{~L}$} & $\mathrm{cCpE}^{\text {wildtype }}$ & ${ }^{192}$ STDIEKEILDLAAATERLN ${ }^{210}$ \\
\hline & $\mathrm{cCpE}^{\mathrm{Ala} 199-202}$ & ${ }^{192} \mathrm{STDIEKE} A A A \mathrm{AAATERLN}{ }^{210}$ \\
\hline \multirow{2}{*}{3} & $\mathrm{cCpE}^{\text {wildtype }}$ & ${ }^{260}$ DFNIYSNNFNNLVKLEQ $^{276}$ \\
\hline & $\mathrm{cCpE}^{\mathrm{Ala} 267-270}$ & ${ }^{260}{ }^{2 F N I Y S N} A A A A$ LVKLEQ $^{276}$ \\
\hline \multirow{2}{*}{4} & $\mathrm{cCpE}^{\text {wildtype }}$ & ${ }^{260}$ DFNIYSNNFNNLVKLEQ ${ }^{276}$ \\
\hline & $\mathrm{cCpE}^{\text {Ala271-272/274-275 }}$ & ${ }^{260}$ DFNIYSNNFNN $A A \mathrm{~K} A A \mathrm{Q}^{276}$ \\
\hline
\end{tabular}

Table 3: Affinities and Kinetics of COP Binding to Claudin-4/cCpE ${ }^{\text {mutant }}$ Complexes

\begin{tabular}{|c|c|c|c|c|}
\hline $\mathrm{COP}$ & claudin-4/cCpE $\mathrm{E}^{\text {mutant }}$ & $k_{\text {on }}(1 / M s)$ & $k_{\text {off }}(1 / s)$ & $K_{D}(n M)$ \\
\hline \multirow{12}{*}{ COP-2 } & \multirow{2}{*}{$\mathrm{cCpE}^{\text {wildtype }}$} & $1.5 \times 10^{4}$ & $7.8 \times 10^{-4}$ & 52.3 \\
\hline & & $\pm 0.1 \times 10^{4}$ & $\pm 0.6 \times 10^{-4}$ & \pm 3.0 \\
\hline & \multirow{2}{*}{$\mathrm{cCpE}^{1}$} & $7.7 \times 10^{3}$ & $1.2 \times 10^{-3}$ & 161.3 \\
\hline & & $\pm 0.5 \times 10^{3}$ & $\pm 0.1 \times 10^{-3}$ & \pm 0.6 \\
\hline & \multirow{2}{*}{$\mathrm{cCpE}^{2}$} & $3.9 \times 10^{4}$ & $3.3 \times 10^{-4}$ & 8.6 \\
\hline & & $\pm 0.5 \times 10^{4}$ & $\pm 0.4 \times 10^{-4}$ & \pm 0.1 \\
\hline & \multirow{2}{*}{$\mathrm{cCpE}^{2 \mathrm{~L}}$} & $1.7 \times 10^{4}$ & $1.0 \times 10^{-3}$ & 57.2 \\
\hline & & $\pm 0.1 \times 10^{4}$ & $\pm 0.1 \times 10^{-3}$ & \pm 3.7 \\
\hline & \multirow{2}{*}{$\mathrm{cCpE}^{3}$} & $1.3 \times 10^{4}$ & $9.4 \times 10^{-4}$ & 64.5 \\
\hline & & $\pm 0.1 \times 10^{4}$ & $\pm 0.1 \times 10^{-4}$ & \pm 4.3 \\
\hline & \multirow{2}{*}{$\mathrm{cCpE}^{4}$} & $5.3 \times 10^{1}$ & $1.3 \times 10^{-2}$ & $252,900.0$ \\
\hline & & $\pm 1.2 \times 10^{1}$ & $\pm 0.1 \times 10^{-2}$ & $\pm 40,700.0$ \\
\hline \multirow{12}{*}{ COP-3 } & \multirow{2}{*}{$\mathrm{cCp} \mathrm{E}^{\text {wildtype }}$} & $2.9 \times 10^{4}$ & $2.7 \times 10^{-3}$ & 98.4 \\
\hline & & $\pm 0.7 \times 10^{4}$ & $\pm 0.5 \times 10^{-3}$ & \pm 11.0 \\
\hline & \multirow{2}{*}{$\mathrm{cCpE}^{1}$} & $1.9 \times 10^{4}$ & $3.9 \times 10^{-3}$ & 200.2 \\
\hline & & $\pm 0.2 \times 10^{4}$ & $\pm 0.6 \times 10^{-3}$ & \pm 5.5 \\
\hline & \multirow{2}{*}{$\mathrm{cCpE}^{2}$} & $11.0 \times 10^{4}$ & $3.0 \times 10^{-3}$ & 24.6 \\
\hline & & $\pm 2.2 \times 10^{4}$ & $\pm 0.1 \times 10^{-3}$ & \pm 4.7 \\
\hline & \multirow{2}{*}{$\mathrm{cCpE}^{2 \mathrm{~L}}$} & $6.3 \times 10^{4}$ & $5.3 \times 10^{-3}$ & 84.4 \\
\hline & & $\pm 0.2 \times 10^{4}$ & $\pm 0.1 \times 10^{-3}$ & \pm 4.8 \\
\hline & \multirow{2}{*}{$\mathrm{cCp}^{3}$} & $3.0 \times 10^{4}$ & $3.9 \times 10^{-2}$ & 1224.6 \\
\hline & & $\pm 0.3 \times 10^{4}$ & $\pm 1.1 \times 10^{-2}$ & \pm 143.7 \\
\hline & \multirow{2}{*}{$\mathrm{cCpE}^{4}$} & $1.0 \times 10^{3}$ & $1.3 \times 10^{-2}$ & $13,300.0$ \\
\hline & & $\pm 0.1 \times 10^{3}$ & $\pm 0.1 \times 10^{-2}$ & \pm 10.0 \\
\hline
\end{tabular}




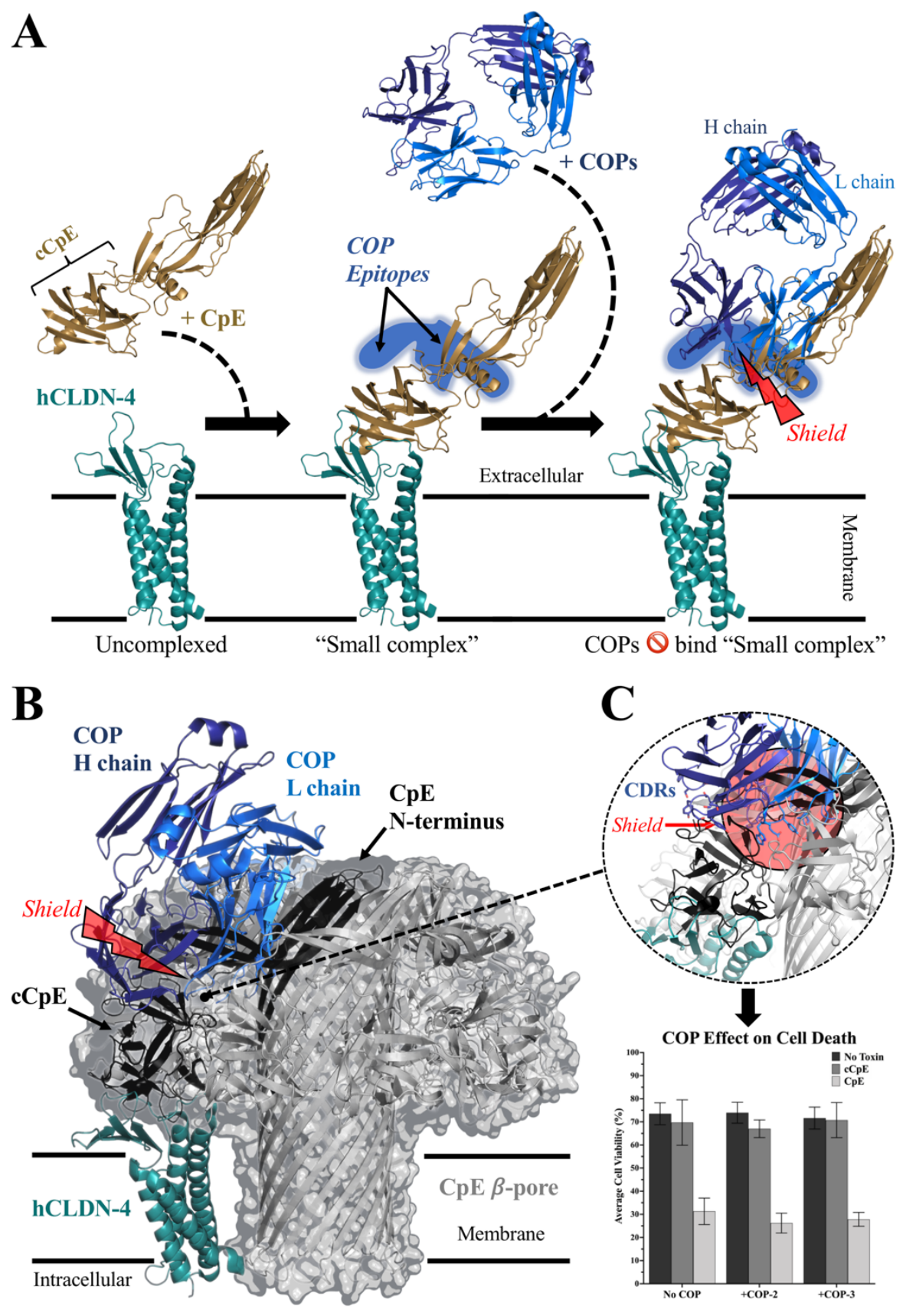

Figure 3. Models for COP Binding to Claudin-4/cCpE and the Claudin-4-bound CpE $\beta$-Pore. (A) $\mathrm{CpE}$ (copper) binding to the ECS of human claudin-4 (hCLDN-4, teal) forms the "small complex". The Nterminus of $\mathrm{CpE}$ sterically shields the $\mathrm{COP}$ (blue) binding epitopes on $\mathrm{c} \mathrm{CpE}$, preventing high-affinity interactions. (B) Model for a $\mathrm{CpE} \beta$-pore (grey) based on the structure of lysenin with one hCLDN-4 (teal) bound to the $\mathrm{cCpE}$ domain of a $\mathrm{CpE}$ monomer (black). Structural rearrangements of the $\mathrm{CpE} \mathrm{N}$-terminus forms the $\beta$-pore. Despite this rearrangement, $\mathrm{CpE}$ multimeric assembly and the $\mathrm{N}$-terminus of $\mathrm{CpE}$ sterically obstruct full COP (blue) engagement with its binding epitopes. (C) Zoom-in on the sterically shielded region shows CDRs from the $\mathrm{L}$ and $\mathrm{H}$ chains cannot access their epitopes on cCpE. Associated bar graph shows the functional effect of COP addition to $\mathrm{CpE}$-induced cytotoxicity of insect cells expressing claudin- 4 on their surfaces. Graph plots the mean and standard deviation based on four readings. Proteins are represented as cartoons (A and $\mathbf{B})$, or as a translucent surface encapsulated cartoon in the case of the $\mathrm{CpE} \beta$-pore (B). 


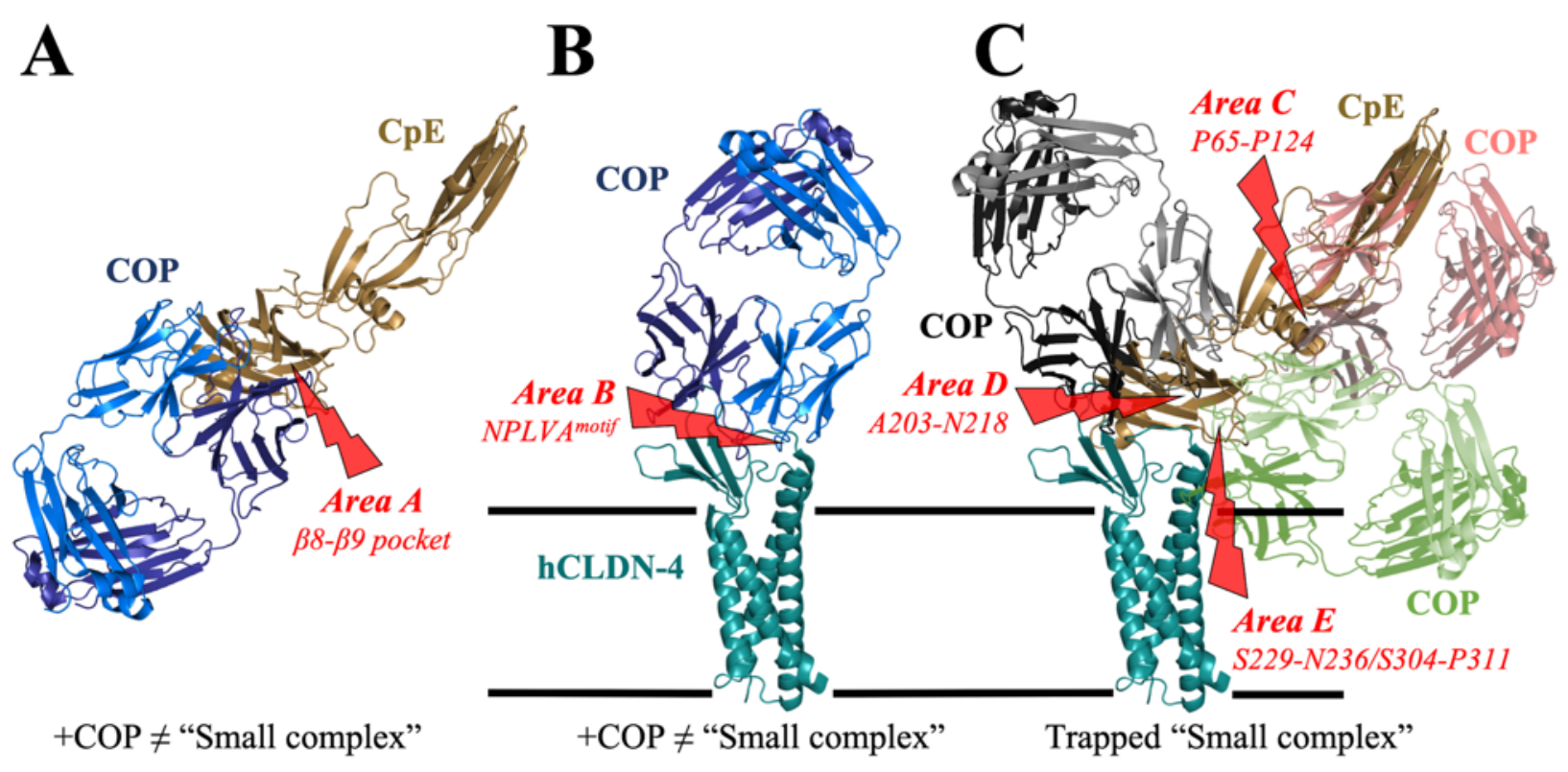

Figure 4. COP Targeting Approaches to Obstruct CpE Cytotoxicity. (A) COPs (blue) that target the $\mathrm{cCpE}$ domain of $\mathrm{CpE}$ (copper) by binding to Area A, a solvent accessible pocket between $\beta 8$ and $\beta 9$ of $\mathrm{cCpE}$ that is known to drive high-affinity binding to claudin-4's NPLVA ${ }^{153}$ motif, which is critical for "small complex" formation. (B) COPs (blue) that target human claudin-4 (hCLDN-4, teal) by binding to Area B, the two ECS of claudins, which both interact with cCpE to initiate "small complex" formation. (C) COPs (red, black, green) that target $\mathrm{CpE}$ (copper) by binding to Areas $C, D$, and E. Area $C$ comprises residues Pro65-Pro124 of CpE's N-terminus, which contains a purported Thr92-Gly105 $\alpha$-helix used for $\beta$-pore formation. Area $D$ comprises residues Ala203-Asn 218 of $\mathrm{cCpE}$, which contains its $\beta 1$ strand and $\alpha 1$ helix. Area $E$ comprises residues Ser229-Asn236 and Ser304-Pro311 of cCpE, which contain two loops that connect strands $\beta 2$ to $\beta 3$ and $\beta 8$ to $\beta$. 


\section{Supplementary Information (SI) Appendix}

Light Chain Alignment

CDR-L

CDR-L2

\begin{tabular}{|c|c|c|}
\hline COP- & 25 & SDIOMTOSPSSLSASVGDRVTITCRASOSVSSAVAWYOOKPGKAPKLLIYSA \\
\hline COP-3 LC & 25 & SDIQMTQSPSSLSASVGDRVT ITCRASQSVSSAVAWYQQKPGKAPKLLIYSASSL \\
\hline BV_ēC & 25 & SDIQMTQSPSSLSASVGDRVTITCRASQSVSSAVAWYQQKPGKAPKLLIYSASSL \\
\hline & & 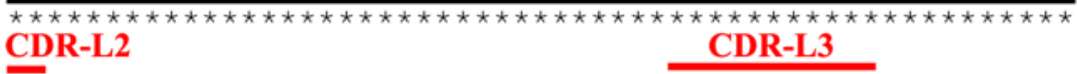 \\
\hline 2 LC & 80 & YSGVPSRFSGSRSGTDFTLTISSLQPEDFATYYCQQSYEW-APVTFGQGTKVE \\
\hline $\mathrm{P}-3^{-} \mathrm{LC}$ & 80 & YSGVPSRFSGSRSGTDFTLTISSLQPEDFATYYCQQSHPWYYP TFGQGTKVEIK \\
\hline BV_ $\bar{L} C$ & 80 & YSGVPSRFSGSRSGTDFTLTISSLQPEDFATYYCQQYLYY-SLVTFGQGTKVEI \\
\hline & & 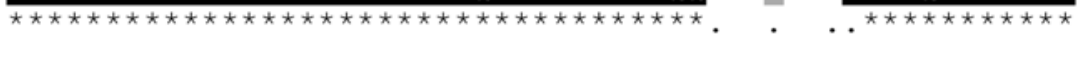 \\
\hline$-2 \_L C$ & 13 & RTVAAPSVFIFPPSDSQLKSGTASVVCLLNNFYPREAKVQWKVDNALQSGNS \\
\hline$-3-L C$ & 135 & RTVAAPSVFIFPPSDSQLKSGTASVVCLLNNFYPREAKVQWKVDNALQSGNSQES \\
\hline$\overline{\mathrm{T}} \mathrm{T}$ & 134 & RTVAAPSVFIFPPSDSQLKSGTASVVCLLNNFYPREAKVQWKVDNALQSGNSQES \\
\hline & & 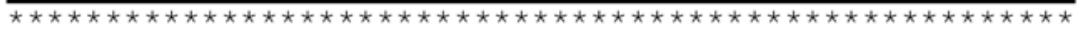 \\
\hline 2_LC & 18 & DSTYSLSSTLTLSKADYEKHKVYACEVTHQGLSSPVTKSFNRGEC \\
\hline 3_LC & 190 & VTEQDSKDSTYSLSSTLTLSKADYEKHKVYACEVTHQGLSSPVTKSFNRGEC \\
\hline$\overline{\mathrm{L}} \mathrm{C}$ & 189 & VTEQDSKDSTYSLSSTLTLSKADYEKHKVYACEVTHQGLSSPVTKSFNRG-- \\
\hline
\end{tabular}

Heavy Chain Alignment

\begin{tabular}{|c|c|c|}
\hline & & CDR-H1 \\
\hline $\mathrm{OP}-2 \mathrm{HC}$ & 24 & SEVQLVESGGGLVQPGGSLRLSCAASGFNESSSS IHWVRQAPGKGLEWVASI \\
\hline $\mathrm{OP}-3_{-}^{-} \mathrm{HC}$ & 24 & VQLVESGGGLVQPGGSLRLSCAASGFNEYSSS IHWVRQAPGKGLEWVAYIS \\
\hline \multirow[t]{2}{*}{ 6CBV_ $\overline{\mathrm{H}} \mathrm{C}$} & 24 & EISEVQLVESGGGLVQPGGSLRLSCAASGFNVVDFS HWVRQAPGKGLEWVAYIS \\
\hline & & 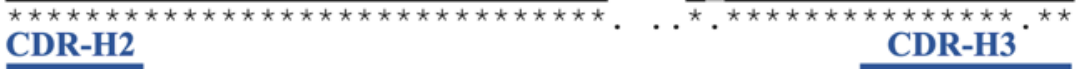 \\
\hline $\mathrm{OP}-2$ - HC & 79 & SYSGYTSYADSVKGRFT ISADTSKNTAYLQMNSLRAEDTAVYYCAR YW SWINSH \\
\hline $\mathrm{OP}-3_{-}^{-} \mathrm{HC}$ & 79 & SYSGYTYYADSVKGRET ISADTSKNTAYLQMNSLRAEDTAVYYCARGY GY DYNF \\
\hline CBV_- & 0 & SSSGSTSYADSVKGRFT ISADTSKNTAYLQMNSLRAEDTAVYYCAR -GY PG-E \\
\hline & & 10 \\
\hline $\mathrm{PP}-2 \mathrm{HC}$ & 134 & YIYSALDYWGQGTLVTVSSASTKGPSVFPLAPSSKSTSGGTAALGCLVKDYFPEP \\
\hline $\mathrm{P}-3^{-} \mathrm{HC}$ & 134 & SVGYALDYWGQGTLVTVSSASTKGPSVFPLAPSSKSTSGGTAALGCLVKDYF PEP \\
\hline CBV_ $\overline{\mathrm{H}} \mathrm{C}$ & 132 & PWWAFDYWGQGTLVTVSSASTKGPSVFPLAPSSKSTSGGTAALGCLVKDYFPEP \\
\hline $\mathrm{PP}-2 \_\mathrm{HC}$ & 189 & VTVSWNSGALTSGVHTFPAVLQSSGLYSLSSVVTVPSSSLGTQTYICNVNHK \\
\hline $\mathrm{P}-3-\mathrm{HC}$ & 189 & VTVSWNSGALTSGVHTFPAVLQSSGLYSLSSVVTVPSSSLGTQTYICNVNHKPSN \\
\hline BV_ & 187 & VTVSWNSGALTSGVHTFPAVLQSSGLYSLSSVVTVPSSSLGTQTYICNVNHKPSN \\
\hline & & \\
\hline $\mathrm{OP}-2 \_\mathrm{HC}$ & 244 & TKVDKKVEPKSCDKTHT \\
\hline $\mathrm{OP}-3_{-}^{-} \mathrm{HC}$ & 244 & TKVDKKVEPKSCDKTHT \\
\hline CBV_ $\overline{\mathrm{H} C}$ & 242 & TKVDKKVEPKS------ \\
\hline
\end{tabular}

SI Figure S1. Sequence Alignment of COPs. The L and H chains of COP-2 and COP-3 were sequenced and aligned against the sequence of a generic sFab, PDB ID 6CBV, using T-Coffee (51). Highlighted in the sequences are the the three CDRs from the L chain (red) and three CDRs from the H chain (blue). 

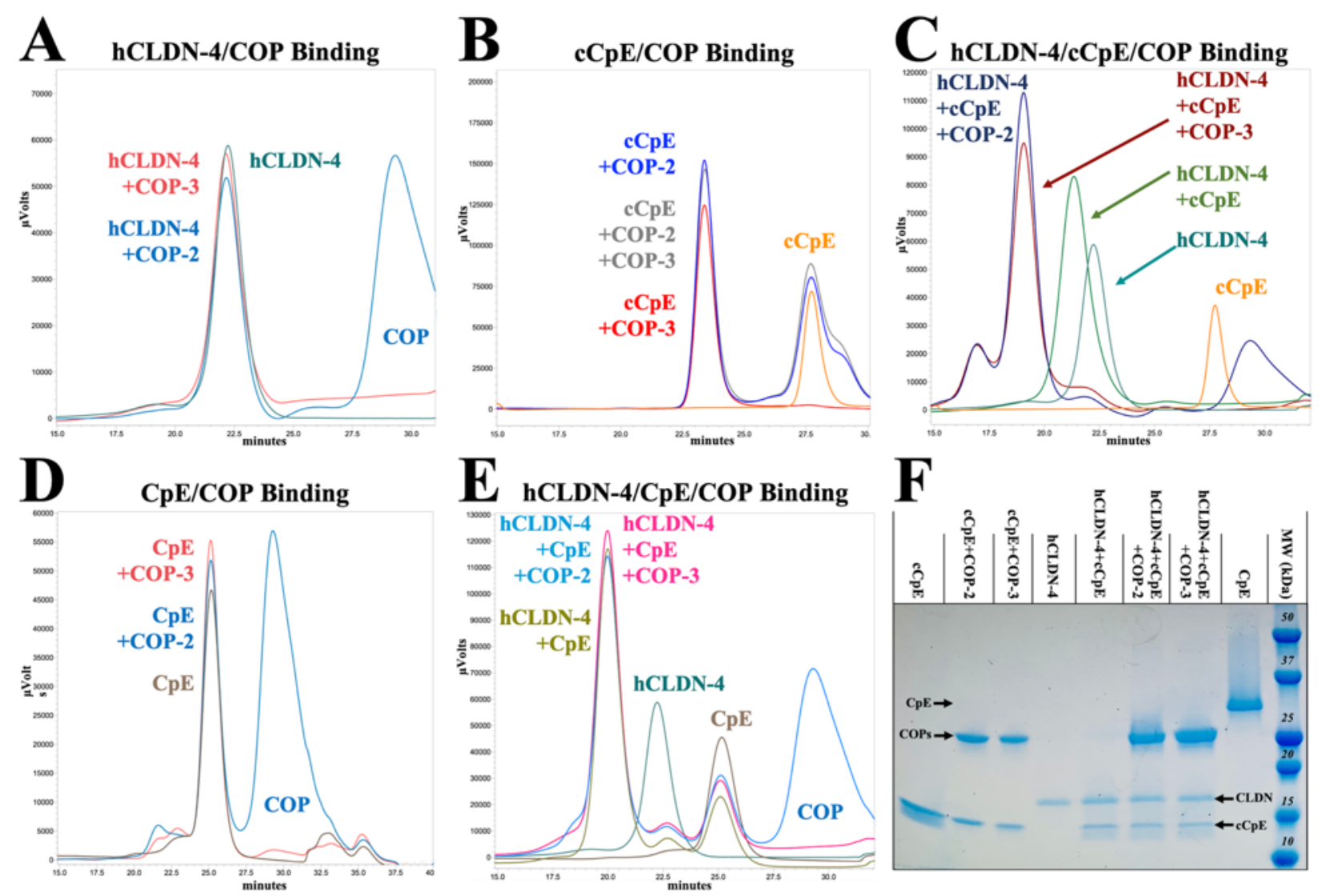

SI Figure S2. Biochemical Characterization of COP Molecular Recognition. Human claudin-4 (hCLDN-4), cCpE, CpE, and COP-2 or COP-3 were incubated together and then injected onto a SEC column equilibrated in DDM and monitoring using $280 \mathrm{~nm}$ absorbance. The SEC traces depict: COP-2 (blue) and/or COP-3 (red) binding to (A) claudin-4 alone (green); (B) cCpE alone (orange); (C) claudin4/cCpE complexes (green); (D) CpE alone (brown); and (E) claudin-4/CpE complexes (brown green). (F) SEC peak fraction from A-E were pooled and subjected to SDS-PAGE. 
A
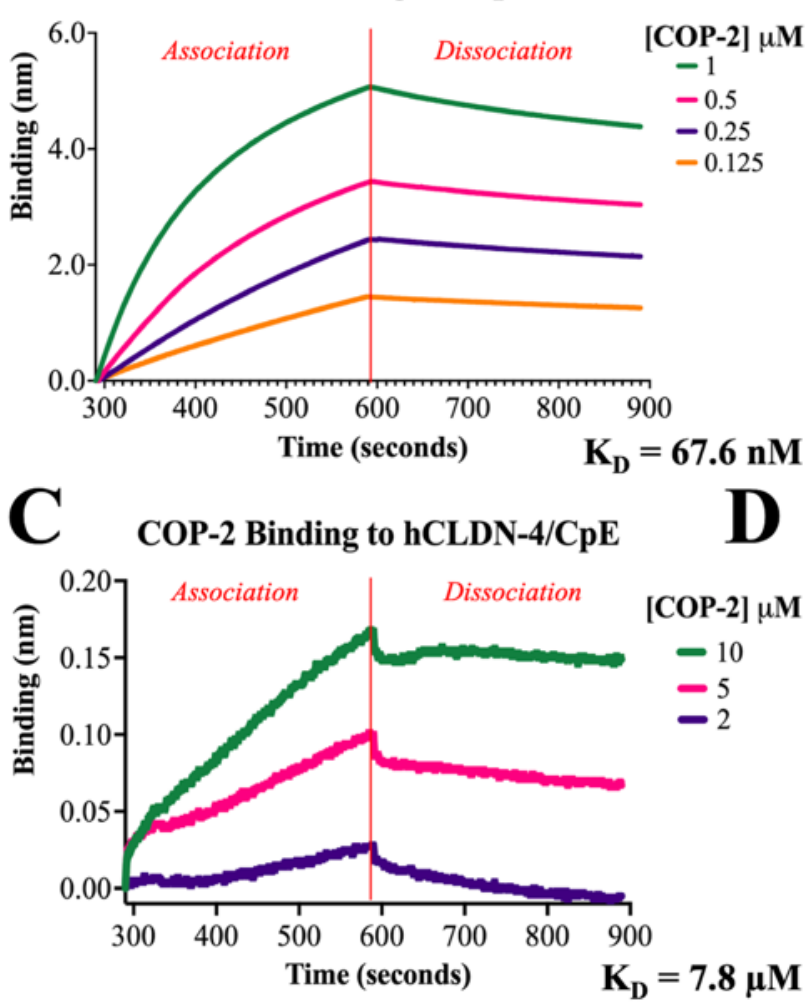

\section{E hCLDN-4 Binding to Mutant $\mathrm{cCpE}^{4}$}

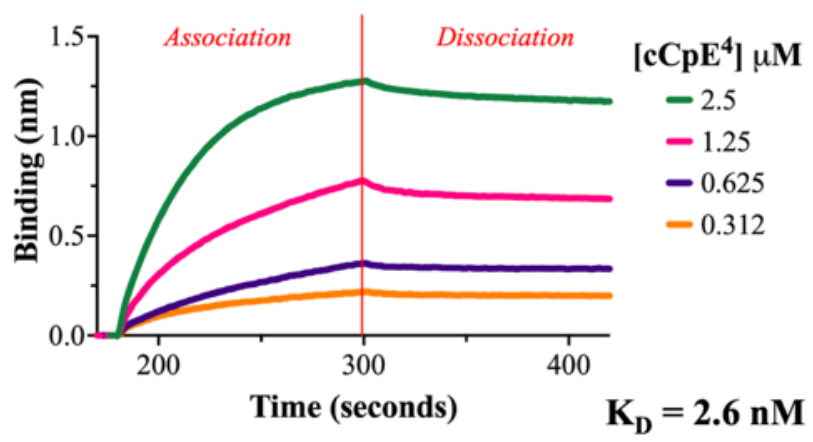

SI Figure S3. Biophysical Binding Measurements Using BLI. (A) COP-2 and (B) COP-3 binding to cCpE-His $_{10}$ immobilized on NiNTA biosensors. (C) COP-2 and (D) COP-3 binding to biotinylated human claudin-4 (hCLDN-4) in complex with cCpE immobilized on streptavidin (SA) biosensors. (E) Mutant $\mathrm{cCpE}^{4}$ binding to biotinylated hCLDN-4 immobilized on SA biosensors. Analyte concentrations are colored from high to low (green, magenta, purple, orange) and a red solid line depicts the transition from association to dissociation phase. BLI sensorgrams collected using BLItz Pro 1.3 Software were re-plotted in Prism 9. Sensorgrams represent a single experiment set from duplicate measurements. 

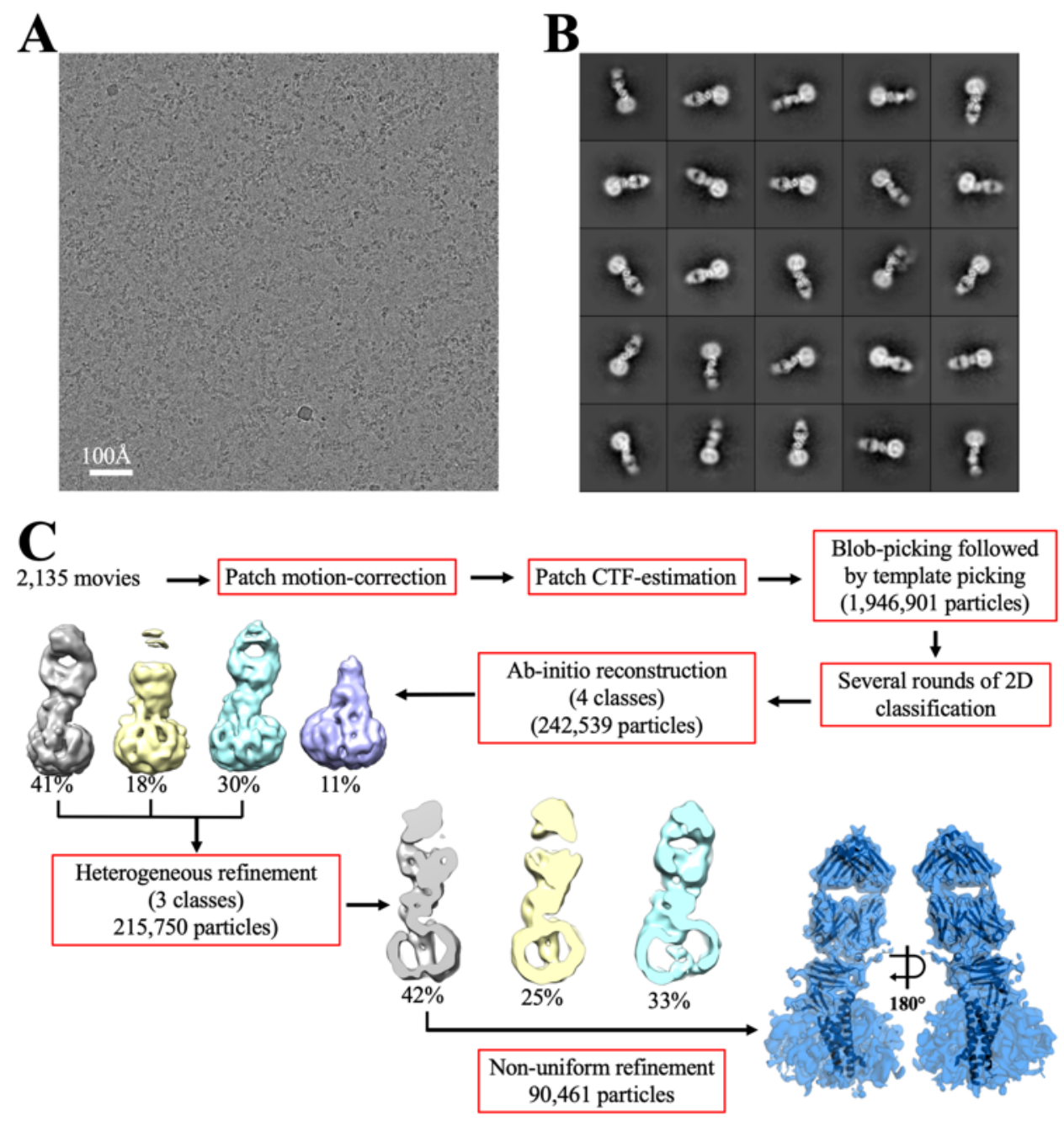

D

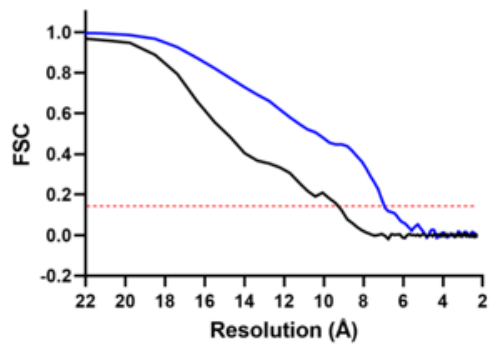

$\mathbf{E}$

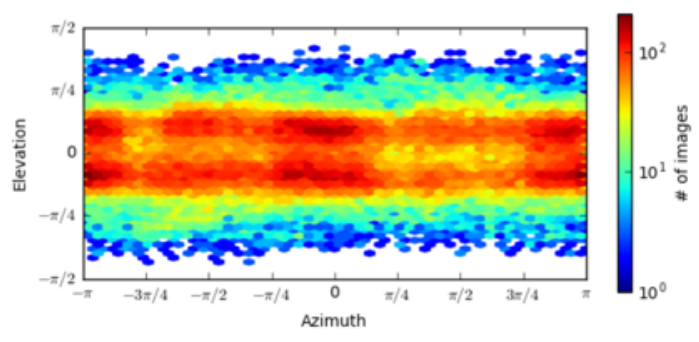

SI Figure S4. CryoEM Data Processing for Claudin-4/cCpE/COP-2 Complexes. (A) Electron micrograph showing the distribution of claudin- $4 / \mathrm{cCpE} / \mathrm{COP}-2$ complexes in ice. (B) Representative 2D class averages of claudin-4/cCpE/COP-2 complexes showing the clear binding of the COP-2 sFab, and signal for TMs within the LMNG detergent micelle. (C) CryoEM data processing workflow. (D) Fourier Shell Correlation (FSC) curves from gold-standard refinement. The $0.143 \mathrm{FSC}$ cutoff is indicated by a dashed red line. The black line indicates FSC without a mask applied, and the blue line indicates FSC with a tight mask applied around the protein and detergent micelle. (E) Angular distribution of particles in the final refinement, demonstrating an absence of pure top views. 
SI Table S1. CryoEM Data Collection, Refinement and Validation Statistics

\begin{tabular}{|c|c|c|c|}
\hline & $\begin{array}{c}\text { Claudin-4/cCpE/ } \\
\text { COP-2 } \\
\text { EMDB-25834 } \\
\text { PDB ID 7DTM } \\
\end{array}$ & $\begin{array}{c}\text { Claudin-4/cCpE/ } \\
\text { COP-3 } \\
\text { EMDB-25835 (whole }) \\
\text { PDB ID 7DTN } \\
\end{array}$ & $\begin{array}{c}\text { Claudin-4/cCpE/ } \\
\text { COP-3 } \\
\text { EMDB-25836 (focused) }\end{array}$ \\
\hline \multicolumn{4}{|l|}{ Data Collection and Processing } \\
\hline Magnification & 92,000 & 120,000 & 120,000 \\
\hline Voltage (keV) & 200 & 200 & 200 \\
\hline Electron exposure $\left(\mathrm{e}-/ \AA^{2}\right)$ & 32.0 & 39.6 & 39.6 \\
\hline Defocus range $(\mu \mathrm{m})$ & $0.8-2.6$ & $1.0-2.2$ & $1.0-2.2$ \\
\hline Pixel size $(\AA)$ & 1.12 & 0.871 & 0.871 \\
\hline Symmetry imposed & $\mathrm{C} 1$ & $\mathrm{C} 1$ & $\mathrm{C} 1$ \\
\hline Number of micrographs & 2,135 & 5,132 & 5,132 \\
\hline Initial particle images (no.) & $1,946,901$ & $3,758,631$ & $3,758,631$ \\
\hline Final particle images (no.) & 90,461 & 305,927 & 305,927 \\
\hline Map resolution $(\AA)$ & 6.9 & 5.0 & 3.8 \\
\hline FSC threshold & 0.143 & 0.143 & 0.143 \\
\hline \multicolumn{4}{|l|}{ Refinement } \\
\hline Initial model used (PDB ID) & $7 \mathrm{KP} 4$ & 7DTM & $N / A$ \\
\hline Model resolution $(\AA)$ & 6.1 & 4.5 & $N / A$ \\
\hline FSC threshold & 0.143 & 0.143 & $N / A$ \\
\hline Map sharpening $B$ factor $\left(\AA^{2}\right)$ & -500 & -150 & $N / A$ \\
\hline \multicolumn{4}{|l|}{ Model composition } \\
\hline Non-hydrogen atoms & 5695 & 5671 & $N / A$ \\
\hline Protein residues & 750 & 746 & $N / A$ \\
\hline \multicolumn{4}{|l|}{$B$ factors $\left(\AA^{2}\right)$} \\
\hline Protein & 164.03 & 413.26 & $N / A$ \\
\hline \multicolumn{4}{|l|}{ R.M.S. deviations } \\
\hline Bond lengths $(\AA)$ & 0.003 & 0.002 & $N / A$ \\
\hline Bond angles $\left({ }^{\circ}\right)$ & 0.726 & 0.681 & $N / A$ \\
\hline \multicolumn{4}{|l|}{ Validation } \\
\hline MolProbity score & 2.23 & 2.27 & $N / A$ \\
\hline Clashscore & 18.29 & 18.83 & $N / A$ \\
\hline Poor rotamers $(\%)$ & 0.00 & 0.48 & $N / A$ \\
\hline \multicolumn{4}{|l|}{ Ramachandran plot } \\
\hline Favored (\%) & 92.58 & 91.73 & $N / A$ \\
\hline Allowed (\%) & 7.42 & 8.27 & $N / A$ \\
\hline Disallowed (\%) & 0.00 & 0.00 & $N / A$ \\
\hline
\end{tabular}



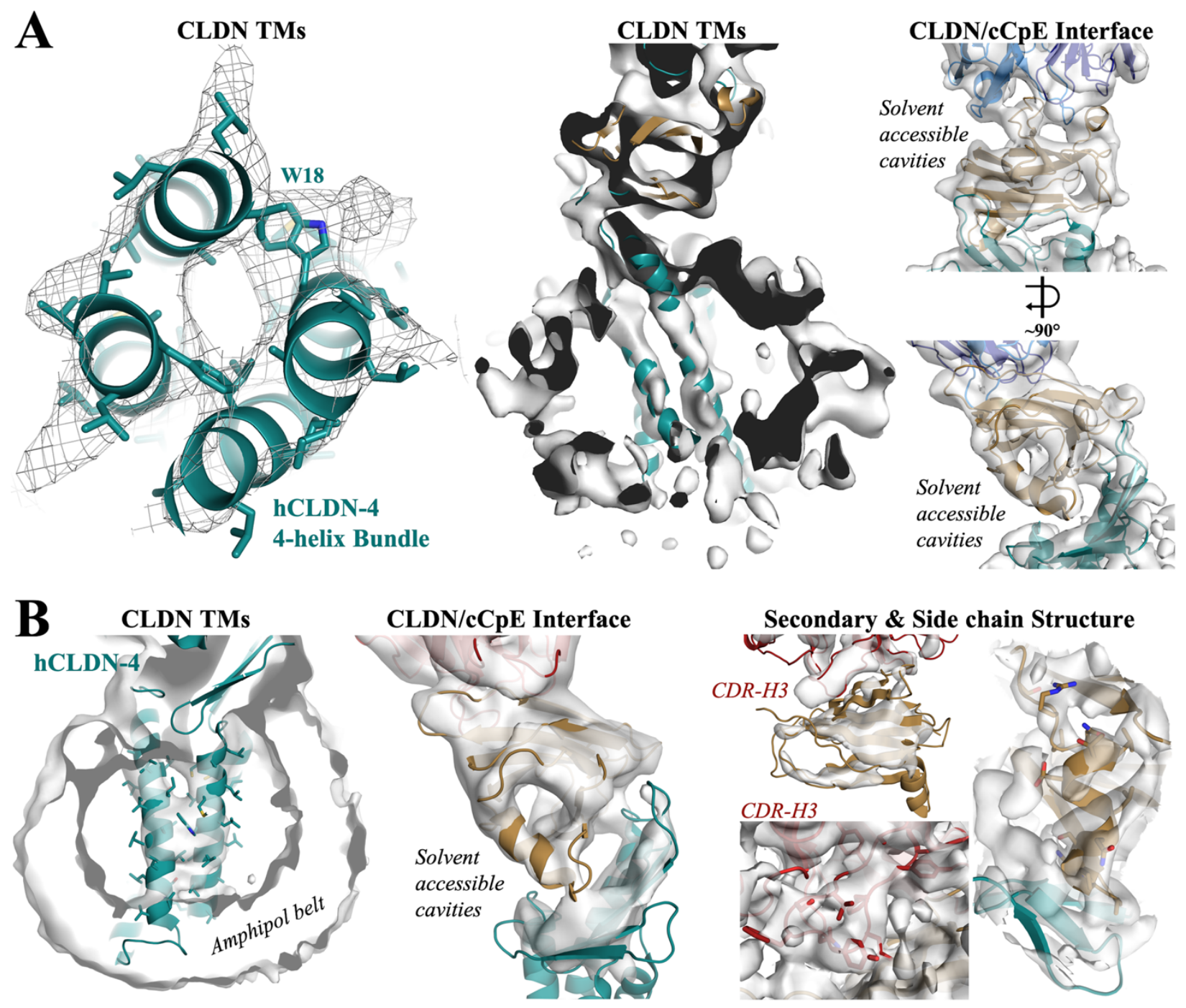

SI Figure S5. CryoEM Map and Structural Features. (A) Structure and corresponding $6.5 \AA$ map from the human claudin-4 (hCLDN-4)/cCpE/COP-2 complex. Images depict: looking up through the TM bundle to the extracellular space with strong side chain density for Trp18 (left); a side view parallel to the membrane plane of individual TMs within the LMNG micelle (middle); and the claudin-4/cCpE and $\mathrm{cCpE} / \mathrm{COP}-2$ interfaces with various solvent accessible cavities resolved (right). (B) Structure and corresponding 4-4.5 $\AA$ map from the claudin-4/cCpE/COP-3 complex. Images depict: a side view parallel to the membrane plane of the TMs within the amphipol belt (left); claudin-4/cCpE interfaces with various solvent accessible cavities resolved (middle); and strand, helix, loop, and side chain density for aromatic amino acids at claudin-4/cCpE and cCpE/COP-3 interfaces (right). Proteins are shown as cartoons and colored as in Fig. 1. 

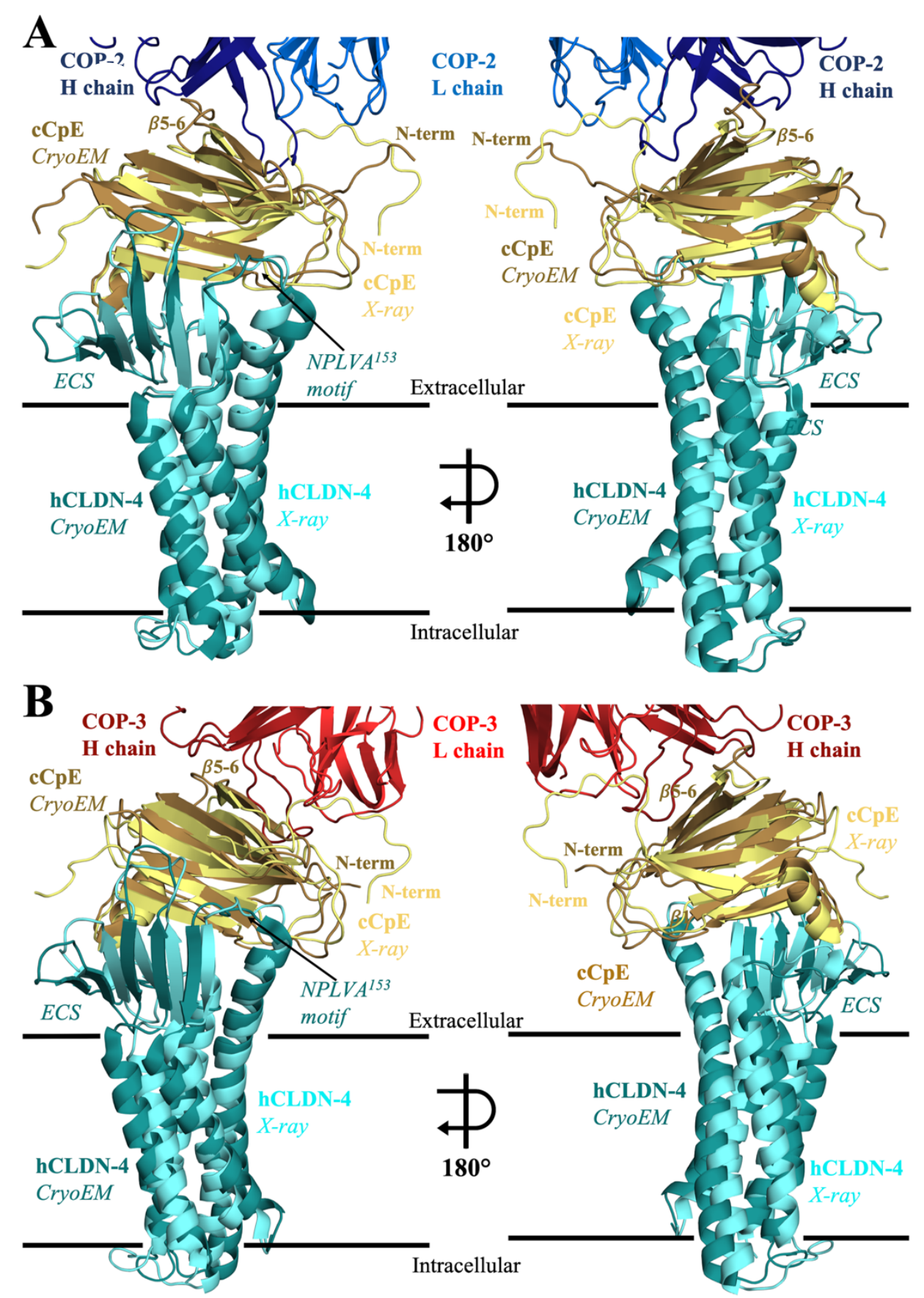

SI Figure S6. Comparison of Claudin-4/cCpE CryoEM and Crystal Structures. Structural overlays depicting similarity between human claudin-4 (hCLDN-4, teal)/cCpE (sand) complexes from cryoEM, and claudin-4/cCpE (cyan/yellow) complex from X-ray crystal structure PDB ID 7KP4. (A) Overlay from COP2-bound (blue) claudin-4/cCpE complex. (B) Overlay from COP-3-bound (red) claudin-4/cCpE complex. Proteins are shown as cartoons with model membrane borders shown as black lines. 
A
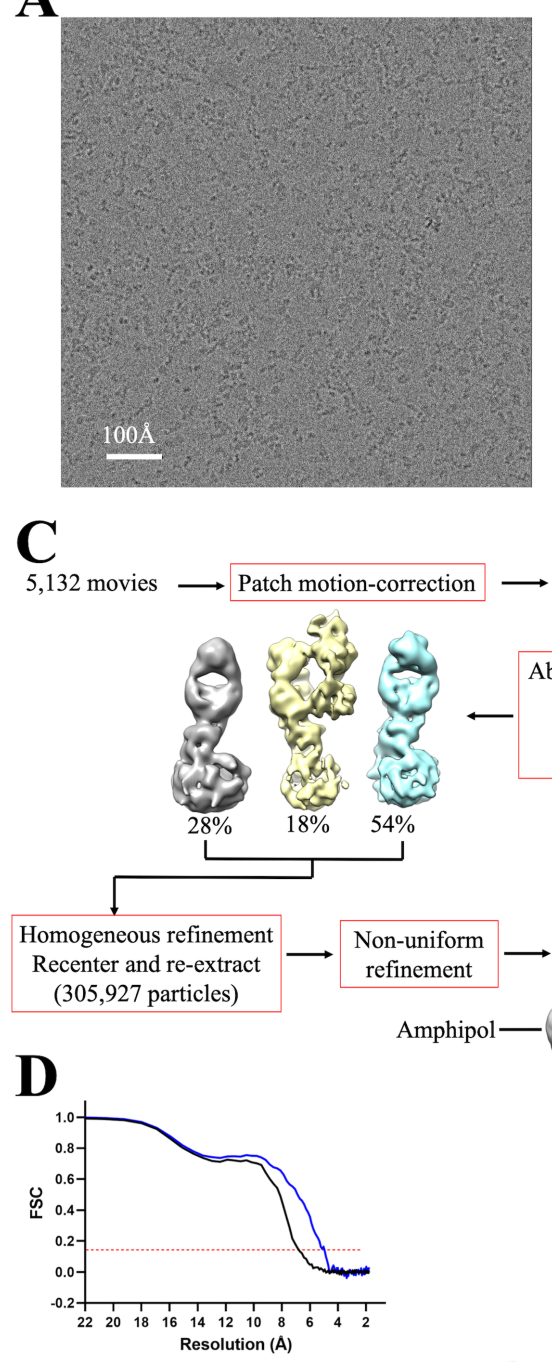

$\mathbf{E}$

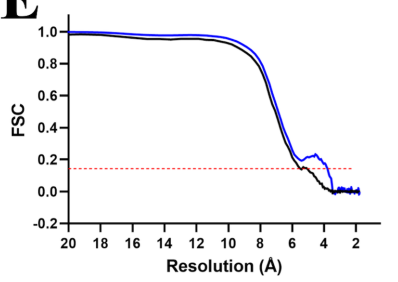
Amphipol-

B
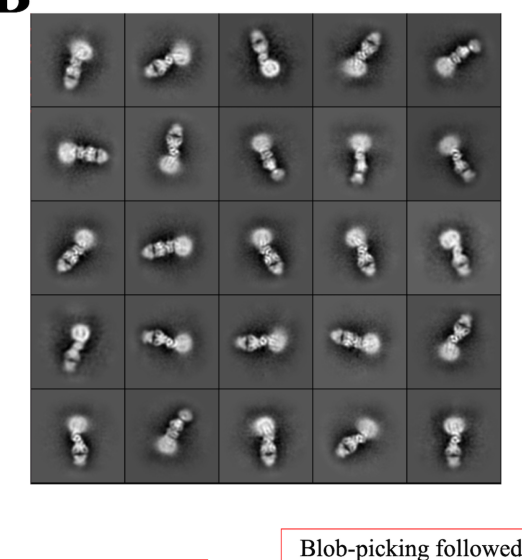
Patch CTF-estimation by template picking
by $(3,758,631$ particles $)$ Several rounds of 2D hetero-refinement ( 3 classes) (379,724 particles)

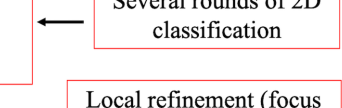

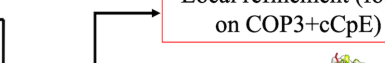
COP-3 $\mathrm{cCpE}$
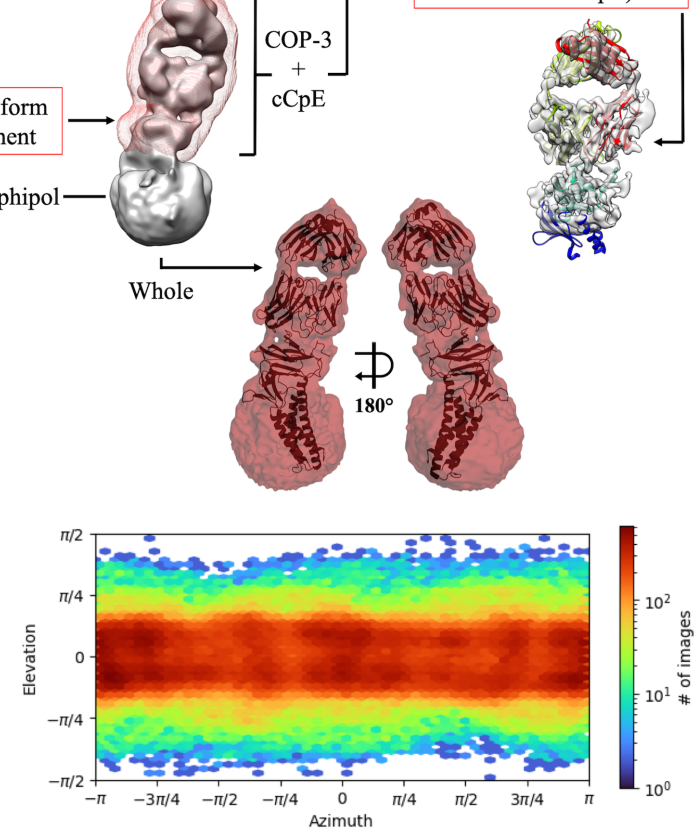

SI Figure S7. CryoEM Data Processing for Claudin-4/cCpE/COP-3 Complexes. (A) Electron micrograph showing the distribution of claudin-4/cCpE/COP-3 complexes in ice. (B) Representative 2D class averages of claudin-4/cCpE/COP-3 complexes showing the clear binding of the COP-3 sFab, and signal for TMs within the amphipol belt. (C) CryoEM data processing workflow. (D) Fourier Shell Correlation (FSC) curves from gold-standard refinement of the entire complex. The 0.143 FSC cutoff is indicated by a dashed red line. The black line indicates FSC without a mask applied, and the blue line indicates FSC with a tight mask applied around the protein and amphipol belt. (E) Fourier Shell Correlation (FSC) curves from local refinement of the $\mathrm{cCpE} / \mathrm{COP}-3$ region. The $0.143 \mathrm{FSC}$ cutoff is indicated by a dashed red line. The black line indicates FSC without a mask applied, and the blue line indicates FSC with a tight mask applied around the $\mathrm{cCpE} / \mathrm{COP}-3$ complex. (F) Angular distribution of particles in the final refinement. 


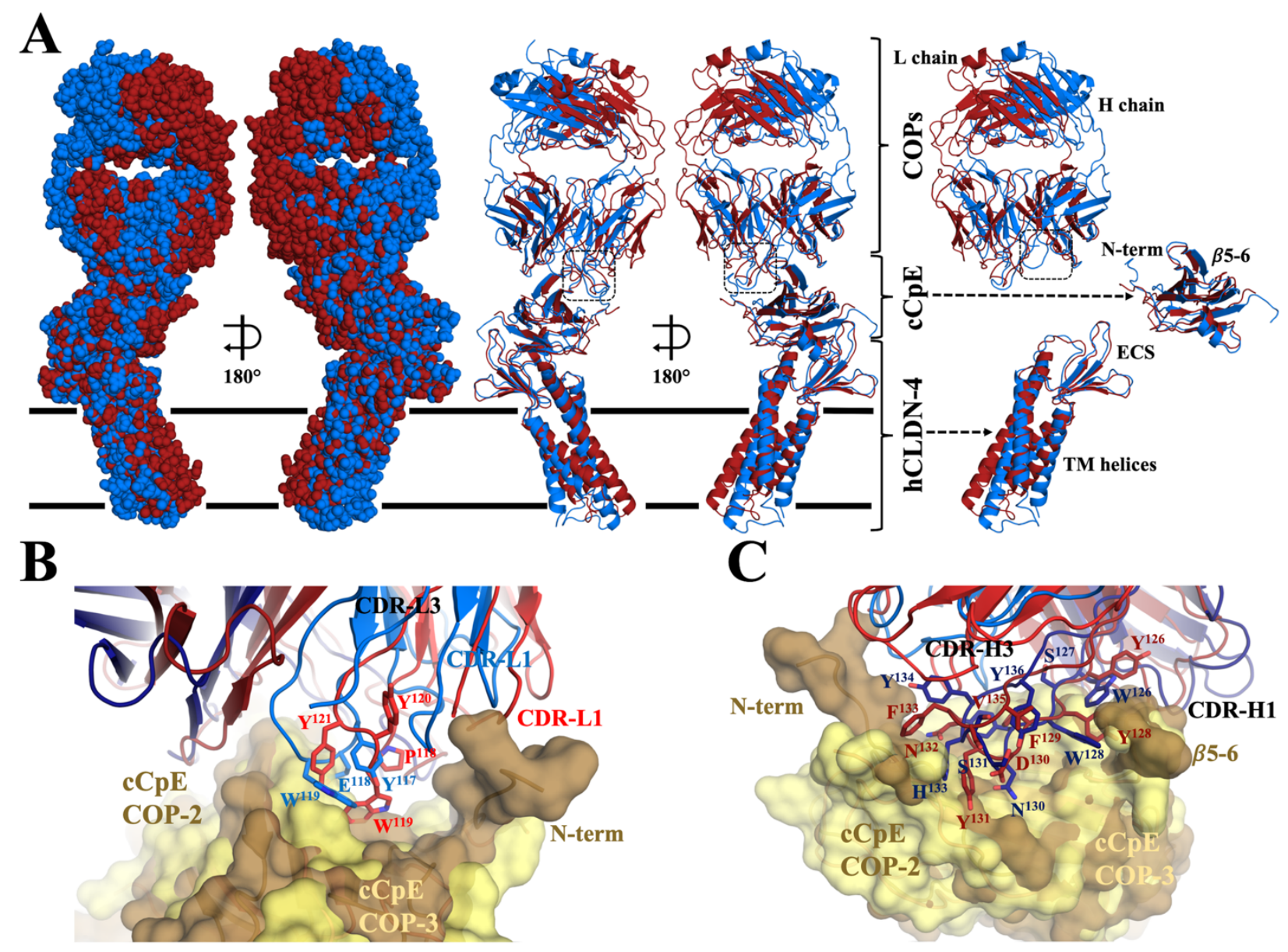

SI Figure S8. Comparison of Claudin-4/cCpE/COP-2 and Claudin-4/cCpE/COP-3 Structures. Structures were overlaid using Chimera (46). (A) Overlay of COP-2-bound (blue) and COP-3-bound (maroon) human claudin-4 (hCLDN-4)/cCpE complexes depicted as a surface (left) or cartoon (middle). Model membrane borders shown as black lines. Overlays of each protein component were made by removing the other two components for ease of visualization and comparison (left). (B) Overlay of COP-2 $\mathrm{L}$ chain (blue) bound to $\mathrm{cCpE}$ (copper) and COP-3 L chain (red) bound to cCpE (yellow). COPs are represented as cartoons while $\mathrm{cCpE}$ is shown as a semi-transparent surface. (C) Overlay of COP-2 H chain (dark blue) bound to $\mathrm{cCpE}$ (copper) and $\mathrm{COP}-3 \mathrm{H}$ chain (maroon) bound to $\mathrm{cCpE}$ (yellow). Proteins are shown as in $\mathbf{B}$. 

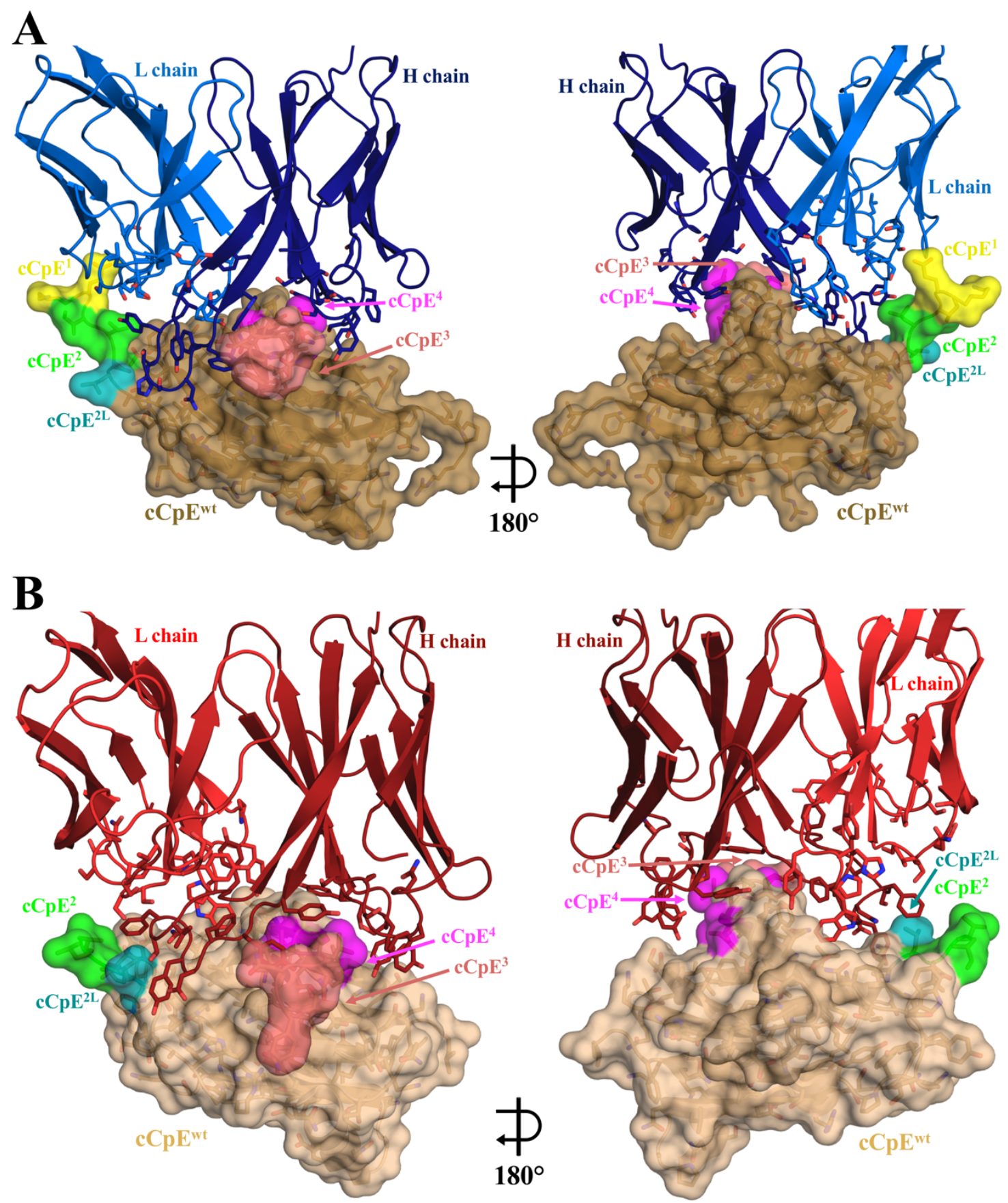

SI Figure S9. Modelled Changes to COP Surface Epitopes for $\mathbf{c C p E}^{\text {mutants }}$. (A) COP-2 (blue) bound to $\mathrm{cCpE}^{\text {wildtype }}$ (copper) with areas of $\mathrm{cCpE}^{\text {mutants }}$ colored as follows: mutant $\mathrm{cCpE}^{1}$ (yellow), $\mathrm{cCpE}^{2}$ (green), $\mathrm{cCpE}^{2 \mathrm{~K}}$ (dark green), $\mathrm{cCpE}^{2 \mathrm{~L}}$ (teal), $\mathrm{cCpE}^{3}$ (salmon), and $\mathrm{cCpE}^{4}$ (magenta). (B) COP-3 (red) bound to $\mathrm{cCpE}^{\text {wildtype }}(\tan )$ with areas of $\mathrm{cCpE}^{\text {mutants }}$ colored as in $\mathbf{A}$. The $\mathrm{cCpE}^{\text {mutants }}$ are visualized as in SI Fig. S8. COPs are shown as cartoons while cCpEs are shown as surfaces. 


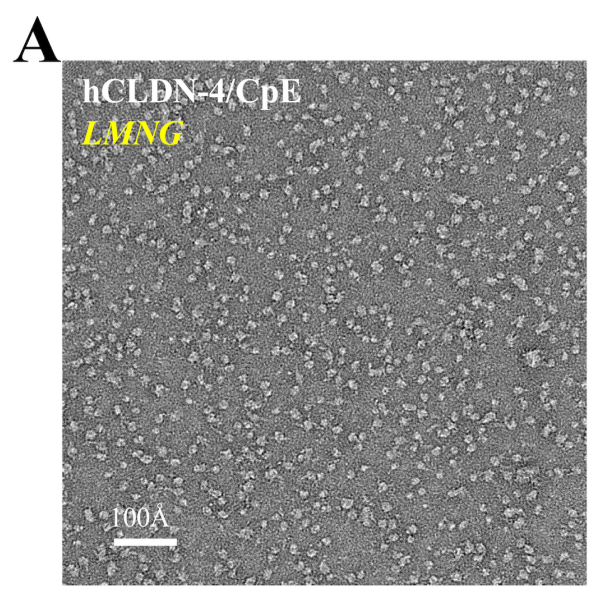

\section{B}
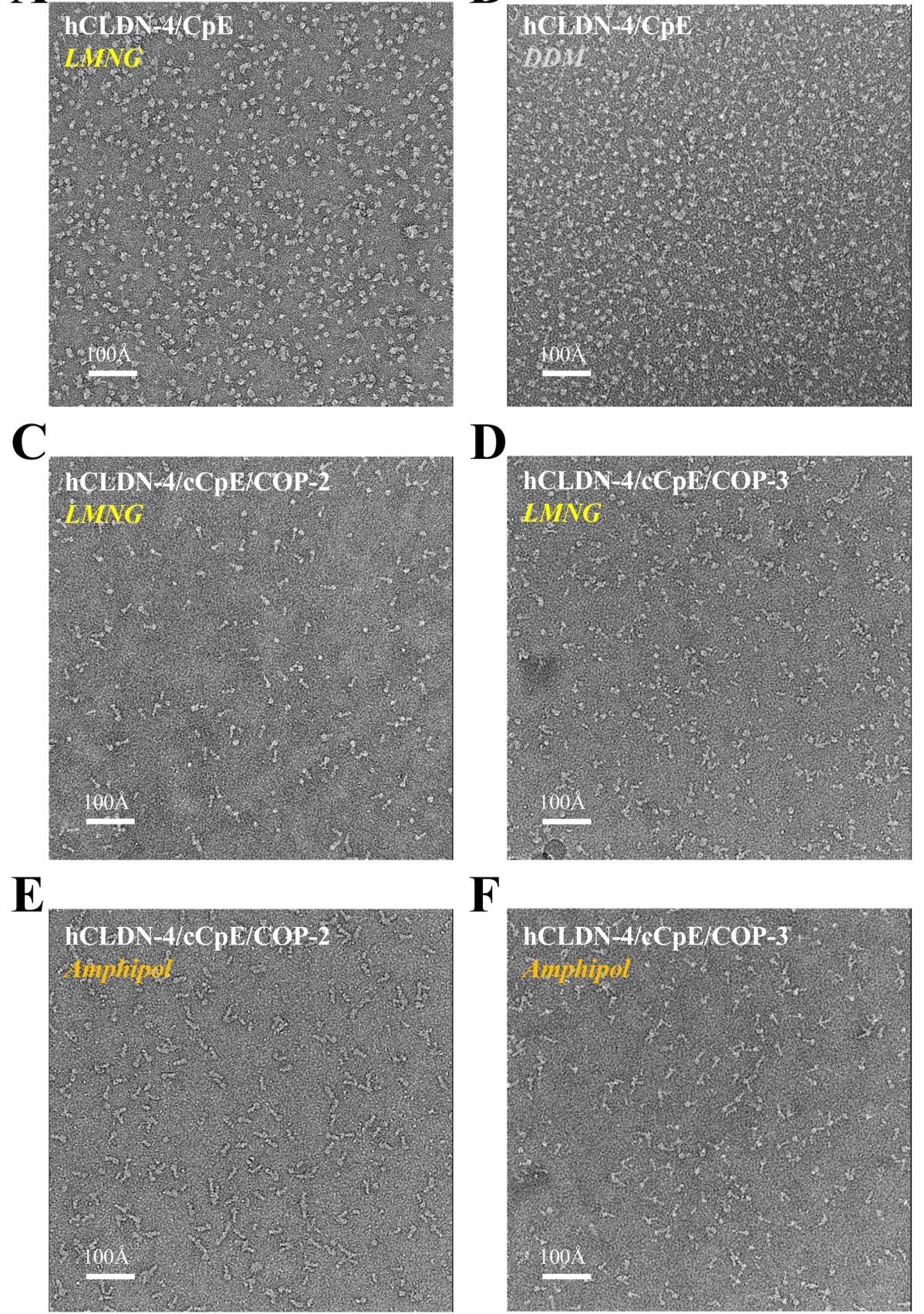

SI Figure S10. Negative Stain EM of Claudin-4/Enterotoxin Complexes in Various Mimetics. (A) Human claudin-4 (hCLDN-4) solubilized in LMNG bound to CpE. (B) hCLDN-4solubilized in DDM bound to CpE. (C) hCLDN-4 solubilized in LMNG bound to cCpE and COP-2. (D) hCLDN-4 solubilized in LMNG bound to cCpE and COP-3. (E) hCLDN-4 solubilized in amphipol bound to cCpE and COP-2. (F) hCLDN-4 solubilized in amphipol bound to cCpE and COP-3. All negative stain data were collected at $200 \mathrm{keV}$ using a screening cryoEM microscope. Samples from panels $\mathbf{C}$ and $\mathbf{F}$ were collected and further processed, resulting in the structures presented here. 